\title{
Chronic neutrophilic leukemia: new science and new diagnostic criteria
}

\author{
Natasha Szuber ${ }^{1}$ and Ayalew Tefferi ${ }^{1}$
}

\begin{abstract}
Chronic neutrophilic leukemia (CNL) is a distinct myeloproliferative neoplasm defined by persistent, predominantly mature neutrophil proliferation, marrow granulocyte hyperplasia, and frequent splenomegaly. The seminal discovery of oncogenic driver mutations in CSF3R in the majority of patients with CNL in 2013 generated a new scientific framework for this disease as it deepened our understanding of its molecular pathogenesis, provided a biomarker for diagnosis, and rationalized management using novel targeted therapies. Consequently, in 2016, the World Health Organization (WHO) revised the diagnostic criteria for CNL to reflect such changes in its genomic landscape, now including the presence of disease-defining activating CSF3R mutations as a key diagnostic component of CNL. In this communication, we provide a background on the history of CNL, its clinical and hemopathologic features, and its molecular anatomy, including relevant additional genetic lesions and their significance. We also outline the recently updated WHO diagnostic criteria for CNL. Further, the natural history of the disease is reviewed as well as potential prognostic variables. Finally, we summarize and discuss current treatment options as well as prospective novel therapeutic targets in hopes that they will yield meaningful improvements in patient management and outcomes.
\end{abstract}

\section{Introduction: "An uncommon myeloproliferative disorder"}

Chronic neutrophilic leukemia $(\mathrm{CNL})$ is a rare $B C R$ $A B L$ negative myeloproliferative neoplasm (MPN) characterized by sustained, predominantly mature neutrophil proliferation, bone marrow granulocytic hyperplasia, and hepatosplenomegaly. It was first described by Tuohy in 1920 in a report entitled "A case of splenomegaly with polymorphonuclear neutrophil hyperleukocytosis" , detailing the case of a 58-year-old woman presenting with splenomegaly and an associated "overwhelming preponderance of mature polymorphonuclear neutrophils". Subsequently, in 1932, Emil-Weil and $\mathrm{See}^{2}$ reported two more possible cases from European literature. Over 30 years later, Jackson and Clark reported on a rare case of MPN, coining the term neutrophilic leukemia ${ }^{3}$, but the inaugural use of the term "chronic neutrophilic leukemia" was finally attributed to Tanzer et al. in the Lancet in

Correspondence: Ayalew Tefferi (tefferi.ayalew@mayo.edu)

${ }^{1}$ Department of Internal Medicine, Division of Hematology, Mayo Clinic, Rochester, Minnesota, USA
$1964^{4}$ and was re-iterated shortly thereafter by Rubin in the Annals of Internal Medicine in $1966^{5}$. Importantly, and perhaps disappointingly, whether these accounts represent actual cases of CNL remains uncertain as literature were scant and diagnostic criteria virtually nonexistent.

As early findings consisted chiefly of isolated case reports or small case series, CNL was born of an era of being defined primarily by what it was not: by excluding basophilia, monocytosis, or a $B C R-A B L$ fusion, it could be distinguished from chronic myeloid leukemia (CML), atypical chronic myeloid leukemia (aCML), and chronic myelomonocytic leukemia (CMML). Similarly, by eliminating potentially confounding underlying clinical conditions such as infections or malignancy it could, sometimes with difficulty, be differentiated from a leukemoid reaction.

Through the years, CNL has regrettably suffered an identity crisis of sorts, even embodying a challenge of nomenclature. In a comprehensive review from 2002 published in the British Journal of Haematology ${ }^{6}$, Reilly 
distinguishes "true CNL" from potential mimickers such as "neutrophilic-chronic myeloid leukemia", or N-CML, "plasma cell dyscrasia-associated neutrophilia", and "myelodysplastic-chronic neutrophilic leukemia," emphasizing the obstacle of identifying cases of diagnostically pure CNL from a virtual diagnostic wastebasket. Even the terms used to describe the disease, themselves, have evolved from "neutrophilic leukemia" to "true chronic neutrophilic leukemia" to simply "chronic neutrophilic leukemia," the current accepted World Health Organization (WHO)-defined diagnostic entity. In his statement: "the literature... is frequently confusing and often incomplete, with the result that CNL's natural history and prognosis remain obscure," Reilly thoughtfully articulated the magnitude of the gap in the state of scientific knowledge of CNL in 2002 and emphasized the need for a more stringent and definitive diagnostic framework ${ }^{6}$.

Accordingly, only a fraction of historically reported cases of "CNL" are actually consistent with present-day diagnostic criteria ${ }^{6}$, and in retrospect, many were probably erroneously labeled CNL when in fact representing cases of reactive neutrophilia or other myeloid malignancies.

The first proposed basic, albeit operational, diagnostic criteria for CNL date back to 1979 in a report by You and Weisbrot ${ }^{7}$. The elements considered central to CNL diagnosis were severe sustained mature neutrophilic leukocytosis, hepatosplenomegaly, absence of leukemoid reaction, and elevated values for leukocyte alkaline phosphatase (LAP), serum vitamin B12, and uric acid. However, it was not until 2001 that the WHO Classification of Neoplastic Diseases formally acknowledged CNL as a distinct myeloproliferative malignancy and included it as an entity in their Proposed WHO Classification of Myeloid Neoplasms ${ }^{8,9}$.

Increasing recognition of this rare MPN along with improvements in the diagnostic approach has prompted a progressive rise in the number of recorded cases of CNL with time. In 1979, only 13 cases had been reported ${ }^{7}$, and by 1996 , there were still fewer than 100 altogether ${ }^{10}$. By 2002, 129 cases of CNL were described in the literature ${ }^{11}$ and by 2005 , there were $150^{12}$. At the present time, over 200 cases of the disease are recorded, although the true occurrence of CNL is likely much lower when strict WHO diagnostic criteria are applied. This has recently been detailed in a review by Bain and Ahmad ${ }^{13}$ counting a total of 52 published cases of "CNL" not meeting WHO diagnostic criteria for the disease.

\section{New science}

The discovery of colony-stimulating factor 3 receptor (CSF3R) mutations in the majority of CNL patients ${ }^{14}$ revolutionized our understanding of the pathogenesis of this disease, provided a biomarker for CNL diagnosis, and paved the way for therapeutic targeting with smallmolecule tyrosine kinase inhibitors (TKI). This landmark finding appropriately marked the beginning of a new era for CNL as it shifted our focus toward genomics at the heart of this once biologically obscure disease. With the subsequent identification of additional genetic lesions involved in CNL oncogenesis, some of which are potentially druggable targets, this molecular revolution in $\mathrm{CNL}$ conclusively marked the birth of new science for this once poorly defined disease entity. In light of these new findings, the aim of this article was to critically review available literature, highlight recent advances in molecular diagnostics, present updated diagnostic criteria, and outline current as well as novel and developing therapeutic approaches.

\section{Epidemiology}

The increasing number of CNL cases being reported has enabled a more accurate assessment of its epidemiology; however recognizing that many of these represent "mimickers" and considering the still relatively scarce literature, the epidemiology of the disease remains imperfectly defined. According to the Surveillance, Epidemiology, and End Results (SEER) Program of the National Cancer Institute, CNL remains a rare disease and its true incidence is unknown ${ }^{15}$. It typically presents in elderly patients, with $88 \%$ of CNL patients being over age 50 in one report ${ }^{11}$. The median age at diagnosis was 66.5 years (range: 15-86) in one of the largest series of 40 WHO-defined CNL cases ${ }^{16}$. Curiously, a recent publication by Ouyang et al. found a much younger median age of 39 years (range 27-92 years) in their 10-patient CNL cohort $^{17}$ which may be biased by a small sample size. Sex distribution was thought to be equal although more recent data shows a slight male preponderance of $56-57 \%$ as revealed by a series of $40 \mathrm{CNL}$ patients and 14 molecularly defined CNL cases, respectively ${ }^{16,18}$. Interestingly, there is also some evidence for a preponderance of males specifically in the CSF3R-mutated cohort of CNL patients $^{17,19}$.

\section{Clinical features}

$\mathrm{CNL}$ is a clinically heterogeneous disease which may present as incidental neutrophilic leukocytosis on a routine blood count or alternatively with a spectrum of constitutional symptoms including fatigue, weight loss, night sweats, bone pain, easy bruising, pruritus, or gout ${ }^{10}$. The majority of patients are asymptomatic at diagnosis. Of presenting clinical manifestations, fatigue appears to be the most common. In a series of 14 molecularly defined CNL patients, $71 \%$ had antecedent chronic leukocytosis lasting a median of 12.5 months (range 5-84 months) ${ }^{18}$. Clinical examination may reveal splenomegaly (present in $36 \%$ in one series ${ }^{18}$ ) involving primarily red pulp cords 
and sinuses, and/or hepatomegaly, but as in CML, lymph node involvement remains relatively uncommon ${ }^{20}$.

There is substantial evidence for an association of CNL with a hemorrhagic diathesis. Reports as early as 1979 describe an "unexplained hemorrhagic tendency" in a portion of CNL patients ${ }^{7}$ which has been reiterated in later reports ${ }^{11,21-23}$, including a considerable number of cases of cerebral hemorrhage. In a report by Bohm, of 14 new cases of CNL reviewed of which 10 had died (mean survival time 14.7 months), the most frequent cause of death was in fact cerebral hemorrhage, seen in six cases ${ }^{11}$. Mechanistically, this bleeding diathesis has been attributed to thrombocytopenia, platelet dysfunction, and/or potentially to vascular wall infiltration by neoplastic cells $^{22,24}$. It has been suggested that patients displaying bleeding complications out of proportion to their platelet count be evaluated for acquired von Willebrand's disease and other acquired coagulation and platelet function disorders.

\section{Laboratory findings}

The hallmark of CNL is sustained, chronic, mature neutrophilia. In a series of WHO-defined CNL, the median leukocyte count was $39 \times 10^{9} / \mathrm{L}$ (range: $11-126$ ) with predominant neutrophilia ${ }^{12}$. Importantly, CNL is characterized by the presence of more mature granulopoietic forms compared with CML, with granulocytes nearly exclusively $(\geq 80 \%)$ at the segmented and band stage of development and minimal to no circulating blasts. The notable absence of monocytosis, basophilia, and eosinophilia is another feature of CNL. The majority of CNL patients present with mild anemia (median hemoglobin $\sim 11 \mathrm{~g} / \mathrm{dL})^{12,16}$ and/or thrombocytopenia. More commonly, thrombocytopenia is reported with disease progression along with increasing splenomegaly, often heralding blast crisis ${ }^{12}$. Lactate dehydrogenase (LDH) levels are typically elevated, as is the LAP score; this latter observation remains non-specific but contrasts with the low LAP score noted in CML. Vitamin B12 levels are also often elevated due to transcobalamin release from granulocytes and their precursors ${ }^{10}$. Interestingly, low serum granulocyte-colony stimulating factor (G-CSF) levels have been documented in CNL patients by several groups ${ }^{25-27}$ though this test is not readily available.

\section{Bone marrow morphology}

Bone marrow is uniformly and intensely hypercellular in CNL due to expanded neutrophilic granulopoiesis with an increased myeloid to erythroid ratio which may be in excess of $20: 1^{28}$. However, the left shift is distinctly more subtle and mature neutrophils more prominent than in CML with the majority of granulocyte precursors being at the metamyelocyte to segmented stages of maturation. Fewer than 5\% myeloblasts are present and there is an absence of Auer rods as well as no dysplastic features ${ }^{16}$. Reticulin staining, though characteristically normal, may show minimal fibrosis but should not exceed a grade of 1 $+{ }^{12}$. Erythroid maturation is normoblastic and megakaryocytes, while morphologically normal, may be normal or slightly increased in number ${ }^{16}$.

\section{Neutrophil morphology}

Neutrophil toxic granulations and Dohle bodies are a common finding in CNL and may be suggestive of an activated neutrophil state. It is of note that these same findings are more frequently observed in neutrophilic leukemoid reactions but remain non-specific ${ }^{13}$. While some reports have shown normal neutrophil chemotaxis in $\mathrm{CNL}^{29}$, others demonstrate increased phagocytosis and superoxide production suggesting increased neutrophil function ${ }^{25}$. Paradoxically other studies have shown attenuated intracellular bactericidal activity in $\mathrm{CNL}^{25,30}$. Whether this biological phenomenon translates clinically to an increased propensity for infection in CNL patients is not clear $^{31,32}$.

\section{Genetic predisposition}

To date there is a single report in the literature of a familial case of suspected CNL involving a father and son who both developed the disease ${ }^{33}$. The father had previously been exposed to radiation following the atomic bomb attack on Hiroshima however, and the oncogenetic effect of this remains a potentially confounding pathogenic factor.

\section{Differential diagnosis}

Neutrophilic leukocytosis may underlie a wide variety of diseases, both benign and malignant. Furthermore, the clinical and laboratory features of CNL may overlap with these disorders. Given that an accurate diagnosis of CNL requires exclusion of a number of mimickers, it is critical to consider potentially confounding diagnoses such as reactive neutrophilia/leukemoid reaction, CML, CML-N, MPN/MDS (myeloproliferative neoplasms/myelodysplastic syndromes) overlap disorders such as aCML and CMML, as well as other myeloid neoplasms.

Distinguishing CNL from a leukemoid reaction can be challenging given that both may present with significant neutrophilia, bone marrow hypercellularity, normal cytogenetics, and absence of $B C R-A B L$ fusion gene. WBC count may be more modestly elevated in leukemoid reaction, though there have been some reports of WBC counts up to $100 \times 10^{9} / \mathrm{L}$ in this context ${ }^{34}$. Meticulous history-taking along with a thorough clinical examination to exclude alternate diagnoses, including occult malignancy or infection, and a period of observation is often recommended before formally determining the diagnosis. The demonstration of clonality including identification of 
a CSF3R mutation or other molecular or cytogenetic abnormality is clearly valuable in aligning the diagnosis in favor of CNL.

CML is invariably associated with a $B C R-A B L$ fusion gene and displays a disproportionally higher percentage of myelocytes. CML also commonly presents with basophilia, thrombocytosis, or eosinophilia. While the absence of the Philadelphia chromosome is implicit to CNL diagnosis, a rare form of CML termed neutrophilic-CML, or CML-N, has been described which shares morphological features of CNL, specifically a prominent neutrophilic proliferation ${ }^{35}$. CML-N, however, is characterized by an uncommon $B C R-A B L$ translocation which results in the transcription of an e19/a2 type $B C R-A B L$ messenger RNA yielding a $230-\mathrm{kD} B C R-A B L$ protein $(\mathrm{p} 230)^{12,36}$. The clinical correlate is a lower total WBC count, less severe anemia, less prominent splenomegaly, and blastic transformation occurring much later in CML-N patients ${ }^{35}$. The attenuated phenotype and indolent course of CML$\mathrm{N}$, specifically in patients without additional cytogenetic abnormalities, is now postulated to be due to low p230 mRNA and protein levels ${ }^{37}$. The diagnosis of CNL also requires that molecular testing be negative for defining markers of alternate neoplasms including not only the $B C R-A B L 1$ fusion gene but also rearrangements in PDGFRA/B or FGFR1, characteristic of eosinophilic leukemia.

Atypical CML represents an uncommon and heterogeneous disease with overlapping features of both myeloproliferative and myelodysplastic neoplasms. It is characterized by leukocytosis (neutrophils and precursors comprising $\geq 10 \%$ of leukocytes) and prominent granulocytic dysplasia, useful in distinguishing it from CNL. Atypical CML also does not meet criteria for $B C R-A B L-$ positive CML, demonstrates absence of $P D G F R A / B$ and FGFR1, and presents with minimal basophilia and monocytosis.

CMML is another MPN/MDS overlap disorder whose diagnosis requires persistent ( $\geq 3$ months) peripheral monocytosis $>1 \times 10^{9} / \mathrm{L}$, absence of $B C R-A B L$ fusion or PDGFRA/B rearrangements, fewer than $20 \%$ blasts or promonocytes in blood and bone marrow, and evidence of dysplasia or clonal abnormality (or persistent monocytosis lasting $\geq 3$ months with exclusion of all other causes). The chronic monocytosis and presence of dysplasia in CMML are central in distinguishing it from CNL.

Other myeloid malignancies must also be excluded, including polycythemia vera (PV), essential thrombocythemia (ET), and primary myelofibrosis (PMF) which each display characteristic genetic and morphological features and whose diagnostic criteria are well outlined in the revised 2016 WHO classification of myeloid neoplasms ${ }^{38}$.
Very rarely, paraneoplastic leukocytosis may result from ectopic production of G-CSF by certain solid tumors, mimicking the neutrophilic leukocytosis of CNL. Such cases are described primarily in association with urological malignancies such as renal cell carcinoma ${ }^{39}$ but have also been reported in other tumor types including lung ${ }^{40}$, mesothelium $^{41-43}$, thyroid $^{44}$, sarcoma ${ }^{45}$, adrenal carcinoma ${ }^{46}$, stomach ${ }^{47}$, gallbladder ${ }^{48}$, and malignant melanoma ${ }^{49}$. The prognostic relevance of tumor-associated GCSF production is uncertain, though some authors have suggested it may portend a poorer prognosis owing to GCSF's role in autocrine growth stimulation, resulting in more aggressive tumor growth ${ }^{46}$.

\section{Disease associations}

Several reports have found associations between CNL and other clonal myeloid and lymphoid disorders with the two most commonly described being polycythemia vera and plasma cell disorders. Though the presence of a concomitant clonal hematologic disease may confound the underlying diagnosis of CNL, there is some literature to convincingly support some of these associations.

\section{Polycythemia vera}

There have been multiple reports describing an association between CNL and PV. These have ranged from accounts of concomitant CNL and PV to cases of PV terminating in a CNL phenotype $\mathrm{e}^{26,50-56}$. However, not only were patients exposed to cytoreductive therapy in virtually all cases published, raising the question of whether these cytotoxic agents may have contributed to CNL pathogenesis, but the number of reports is too limited to draw definitive conclusions. Furthermore, in some such cases, patients harbored comorbidities potentially associated with reactive neutrophilia, including concomitant malignancies, challenging the very diagnosis of CNL. Thus, although not all cases of CNL-PV represent true associations, those that do may lend support to the clonal origin of CNL as well as its classification among the MPN.

\section{Plasma cell disorders}

A disproportionately high number of cases of CNL associated with plasma cell neoplasms have been reported considering the rarity of this disease $\mathrm{e}^{57-65}$, even prompting the distinction of a "monoclonal gammopathy-associated CNL" or MG-CNL entity. Plasma cell dyscrasias have been shown to occur in up to $32 \%$ of CNL cases ${ }^{66}$. In a recent review on this subject, after excluding suspected cases of CNL not meeting WHO criteria, ultimately, 49 patients with "CNL" or neutrophilic leukemoid reaction associated with multiple myeloma or MGUS were found in the literature ${ }^{13}$. The associated plasma cell dyscrasia was multiple myeloma in the majority of cases, followed by MGUS and rarely plasmocytoma ${ }^{13}$. The majority of 
patients expressed lambda light chain with the remainder, kappa, and rarely both ${ }^{13}$, supporting previous reports of predominantly lambda light chain restriction in CNLassociated plasma cell dyscrasias ${ }^{63}$.

The fundamental question plaguing this relationship has remained whether the CNL and plasma cell disorders are clonally related or whether the neutrophilia is secondary to the underlying plasma cell neoplasm. Erber and Reilly raised this question in their aptly titled commentary "Chronic neutrophilic leukemia with plasma cell dyscrasia: friends or relatives?" ${ }^{\prime 67}$. Overall, three main hypotheses have emerged: firstly, the possibility of a common progenitor cell differentiating along both myeloid and lymphoid lines resulting in a biphenotypic neoplasm; secondly, the co-existence of two independent clonal disorders; and thirdly, a purely reactive neutrophilia associated with the plasma cell dyscrasia. The aforementioned review by Bain et al. asserts that leukocytosis in suspected concurrent neoplasms is attributable, in the majority of cases, to cytokine-driven neutrophilia accompanying the primary plasma cell neoplasm ${ }^{13}$ and there is substantial evidence corroborating this, including demonstration of high G-CSF levels and neutrophil counts in a patient with myeloma whose levels declined upon treatment with steroids ${ }^{68}$. Moreover, several other studies have confirmed G-CSF synthesis by neoplastic plasma cells ${ }^{69-74}$. Furthermore, there are reports supporting the polyclonal nature of the plasma cell dyscrasiaassociated neutrophilia, cases of spontaneous remission of the presumed CNL, and even improvements in neutrophil count and hepatosplenomegaly following treatment of the underlying dysproteinemia ${ }^{62,63,72,75}$. Another important distinction is a prognostic one as MGUS-associated CNL patients appeared to have superior survival when compared to strictly WHO-defined $\mathrm{CNL}^{75,76}$ suggesting different etiopathogeneses. The fact that Pardanani et al. did not find CSF3R mutations in five cases of plasma cell neoplasm-associated "CNL" also suggests that both entities are distinct ${ }^{76}$. In addition, the fact that MGUS is common within the age range in which CNL characteristically occurs further supports a merely incidental association. As a whole, this data argue in favor of polyclonal neutrophilic proliferation secondary to antecedent or underlying plasma cell disorder. Consequently, any suspected association should be confirmed by demonstrating neutrophil clonality.

Challenging this precept, Nedeljkovic et al. provided evidence that the neutrophilia in one case of paraproteinemia in a patient with CNL was clonal and not reactive $^{77}$. This individual harbored a JAK2V617F mutation, establishing the clonal nature of the myeloid proliferation. Another similar case of JAK2V617F-positive CNL with MGUS was subsequently reported ${ }^{76}$. There is also an account of emergence of CSF3R mutations during follow-up of a patient with MGUS, representing the first case of a plasma cell neoplasm associated with CNL as defined by this highly sensitive and specific clonal mar$\mathrm{ker}^{78}$. More recently, SETBP1 mutations (D868N and G870S) were identified in two of five cases of neutrophilia with a concomitant plasma cell neoplasm, also suggesting a separate clonal myeloid entity in these individuals ${ }^{79}$. These cases thus support clonal neutrophilia concurrent with plasma cell dyscrasia in at least a portion of described reports based on the critical finding of a clonal myeloid marker.

Altogether, though the nature of the putative CNLdysproteinemia relationship remains controversial, it is likely that both clonal and non-clonal neutrophilia are possible in association with plasma cell neoplasms. The WHO once recommended that all suspected cases of CNL be screened for plasma cell disorders ${ }^{28}$ and that if present, neutrophil clonality be demonstrated via cytogenetic or molecular techniques in order for the hypothesis of CNL to be viable; however, with the current availability of the sensitive and specific CSF3R marker, as will be discussed, this is no longer required.

\section{Other associations}

Rare reports have shown CNL evolving from $\mathrm{MDS}^{80}$ as well as an association with chronic lymphocytic leukemia $^{81}$ but these accounts remain anecdotal. More recently, Wang et al. reported a case of biopsy-proven nephrotic syndrome (kidney biopsy positive for $J A K 2 \mathrm{~V} 617 \mathrm{~F}$ ) related to JAK2-positive CNL successfully treated with Hydroxyurea followed by Hydroxyurea combined with Interferon ${ }^{82}$.

\section{Molecular cytogenetics and clonality}

Evidence for clonality in CNL was initially supported by the finding of a monoclonal methylation pattern of the Xlinked hypoxanthine phosphoribosyl transferase (HPRT) gene in a patient with $\mathrm{CNL}^{83}$. Other groups subsequently demonstrated the clonal nature of the leukemic neutrophils in CNL using HUMARA clonality studies ${ }^{84}$ and fluorescent in situ hybridization (FISH) ${ }^{85}$. Although the majority of patients with CNL display normal cytogenetics at diagnosis ${ }^{12,28}$ the demonstration of non-specific cytogenetic abnormalities in a proportion of CNL patients served as later proof of clonality.

A report by Didonato et al. was the first to demonstrate cytogenetic aberrations in the form of trisomy 9 and a partial deletion of the long arms of chromosome 20 in a patient with suspected CNL ${ }^{86}$. Ensuing reports confirmed the occurrence of cytogenetic abnormalities in a minority of CNL patients at diagnosis and/or during clonal evolution $^{6,12,87}$. In Reilly's 2002 review, 37\% of CNL cases presented abnormal cytogenetics consisting primarily of trisomy 8 , trisomy 21 , deletion $11 \mathrm{q}$, and deletion $20 \mathrm{q}^{6}$. 
Subsequently one of the largest CNL case series (total of 40 CNL patients) identified cytogenetic abnormalities in 13 of 40 patients $(32.5 \%)^{12}$. These aberrations were detected at baseline in $20 \%$ of patients and during clonal evolution in the remaining $12.5 \%$ and included deletion 20q, trisomy 21 , deletion $11 \mathrm{q}$, and deletion $12 \mathrm{p}^{12}$. Though the most common chromosomal lesions in CNL consist of trisomy 8 and deletion 20q, observed either at diagnosis or at the time of clonal evolution ${ }^{18}$, multiple additional abnormalities have been described including tetraploidy 21, trisomies 7, 8, and 9, translocation 1;20, deletion $\mathrm{Y}$, deletion 6 , add 5 p, deletion 15 , and monosomy $2^{88}$, and are considered non-specific, yet non-random findings in myeloid neoplasms ${ }^{89}$.

In rare cases, detection of a well-known MPN molecular marker such as $J A K 2 \mathrm{~V} 617 \mathrm{~F}$ may also serve to establish clonality $^{12,90}$.

\section{Molecular pathogenesis and diagnosis CSF3R mutations}

G-CSF is a cytokine growth factor that regulates basal as well as stimulated "emergency" granulopoiesis and promotes granulocyte differentiation ${ }^{91}$. G-CSF is secreted principally by macrophages, fibroblasts, and endothelial cells. The pivotal role of this mitogenic cytokine in granulopoiesis was underscored as CSF3 or CSF3 receptor (CSF3R)-deficient mice were found to be severely neutropenic $^{92,93}$. The G-CSF receptor, CSF3R, is a single chain cell-surface receptor belonging to the cytokine receptor type I superfamily whose gene maps on chromosome $1 \mathrm{p} 34.3$ and consists of 17 exons $^{91}$. Its cytoplasmic domain contains distinct functional regions with the membrane-proximal region being functionally ascribed to a mitogenic signaling function while the carboxy terminal is involved in maturation signaling and regulation/suppression of proliferation ${ }^{94}$.

Upon binding to its receptor, G-CSF exerts its effects via classical downstream pathways Janus kinase (JAK)-signal transducer and activator of transcription (STAT), SRC family kinases (notably LYN) ${ }^{95,96}$, non-receptor tyrosine kinase SYK, Ras/Raf/MAP kinases, and PI3K/Akt pathways, inducing neutrophilic differentiation, proliferation, and survival as well as stimulating neutrophil function ${ }^{97}$. The discovery of the colony-stimulating factor 3 receptor (CSF3R) mutations in the majority of CNL patients represents a turning point in our understanding of the molecular pathogenesis of this disease ${ }^{14}$. Before this seminal finding, however, CSF3R mutations were already recognized in several forms of myeloid disorders including severe congenital neutropenia $(\mathrm{SCN})$, hereditary chronic neutrophilia, and rarely in myeloid leukemias, as will briefly be discussed below.

\section{Severe congenital neutropenia}

SCN is a clinically heterogeneous inherited condition characterized by early onset of severe bacterial infections. Acquired somatic truncating mutations in CSF3R, analogous to those found in $\mathrm{CNL}^{14}$, have been described in up to $30-40 \%$ of patients with $\mathrm{SCN}^{98}$ concurrently with inherited, pathogenically relevant mutations such as ELANE or HAX199. Though these acquired, mostly nonsense CSF3R mutations were initially thought to contribute to SCN pathogenesis ${ }^{100,101}$, they were later demonstrated to molecularly annotate a subset of $80 \%$ of SCN patients at high risk for leukemic conversion ${ }^{98,101-}$ 105. These truncating mutations, which lead to loss of carboxy-terminal regulating domain, are neither required nor sufficient for induction of leukemia in SCN, as supported by knock-in murine models ${ }^{106}$. It is currently postulated that through co-operation with other oncogenes $^{103,107}$, they provide a clonal advantage of prolonged cell survival ${ }^{108}$ favoring the acquisition of additional oncogenic "hits" ultimately resulting in a leukemic phenotype $^{106}$.

Interestingly, in 2012, Beekman et al. reported a novel acquired membrane proximal CSF3R autoactivating mutation, now known to be CSF3RT618I (also referred to as CSF3RT595I if the 23-amino-acid signal peptide is excluded from the numbering of amino acids), in a patient with SCN having developed AML 17 years after initiating G-CSF treatment (in this case co-occurring with a CSF3R truncating mutation $)^{103}$. The same mutation was also reported in one of 199 other AML cases published by the Cancer Genome Atlas ${ }^{103}$, highlighting abnormal G-CSF signaling as a potential driver of leukemic transformation.

\section{Hereditary chronic neutrophilia}

Another distinct CSF3R mutation has been involved in the pathogenesis of hereditary neutrophilia. The germline, autosomal dominant transmembrane domain CSF3RT617N mutation was described by Plo et al. ${ }^{109}$ and was found to induce dimerization of the CSF3R transmembrane domain, promoting constitutional activation of the receptor in a family with chronic neutrophilia (12 affected individuals out of 16 , over 3 generations). The CSF3RT617N mutation induced receptor-independent granulocyte proliferation and downstream constitutive phosphorylation of JAK2, STAT3, AKT, and ERK which was sensitive to JAK2 inhibition ${ }^{109}$. In the affected family, neutrophilia was accompanied by splenomegaly and increased circulating CD34-positive myeloid progenitors, with only the proband progressing to myeloid malignancy $(\mathrm{MDS})^{109}$. This report underscores the importance of seeking out a family history of neutrophilia in suspected cases of CNL in order to exclude a hereditary basis. 


\section{Leukemia}

Incidentally, the same membrane-proximal CSF3RT617N mutation found in hereditary neutrophilia was first described as a possible polymorphism in AML in $1996^{110}$ and later confirmed as a leukemia-specific mutation in two of 555 AML patients; ${ }^{111}$ it is also rarely found in $\mathrm{CNL}^{112}$.

Zhang et al. recently identified a novel activating mutation (W341C) in the extracellular domain of CSF3R in a leukemic patient, coexpressed with a truncation mutation $(\mathrm{W} 791 \mathrm{X})^{113}$. In vitro studies demonstrated W341C-induced constitutive activation of JAK-STAT and ERK, G-CSF independent cell growth, and transformation of BaF3 cells which translated clinically to progressive leukocytosis and thrombocytopenia. The oncogenic mechanism of W341C was attributed to unpaired cysteine-mediated disulfide bond formation leading to CSF3R dimer stabilization ${ }^{113}$.

\section{Pediatric AML}

In recent years, CSF3R mutations have been identified at a rate of $2.4 \%$ in pediatric AML patients and found to exhibit a strong association with CEBPA mutations ${ }^{114}$. In this setting, patients with transforming CSF3R mutations have displayed a trend toward low risk disease, which has been ascribed to their association with the more favorable CEBPA mutations.

\section{CSF3R mutations in CNL: "The CSF3R mutations represent a biologically unifying feature of $\mathrm{CNL}^{114}$}

In 2013, a landmark study by Maxson et al. was published identifying disease-defining oncogenic CSF3R mutations in the majority of patients with $\mathrm{CNL}^{14}$. This report heralded a new era in the history of $\mathrm{CNL}$ as it elucidated its molecular pathobiology, provided a biomarker for diagnosis, and rationalized the therapeutic use of JAK inhibitors. In this study, CSF3R mutations were found in $89 \%$ and $40 \%$ of CNL and aCML cases, respectively. Two mutational variants of CSF3R were identified: the more common membrane proximal mutations consisting primarily of T618I and T615A point mutations, and frameshift or non-sense mutations leading to premature truncation of the cytoplasmic tail of CSF3R (D771fs, S783fs, Y752X, and W791Z). The truncation mutations, which also occur in SCN, typically coexisted with membrane proximal or transmembrane CSF3R mutations as compound mutations ${ }^{14,115}$. Mechanistically, the membrane proximal mutations prevent $O$-glycosylation of CSF3R resulting in increased active dimeric configuration, ligand-independent receptor activation, and subsequent constitutive downstream signaling through JAK2 ${ }^{14,116}$. Alternately, several mechanisms may explain how truncation mutations lead to uncontrolled cellular proliferation. Truncation of the receptor involves a loss of negative regulatory motifs including the dileucine sorting motif, which plays a role in receptor internalization ${ }^{117}$, and binding sites for the suppressor of cytokine signaling 3 (SOCS3) which normally targets the receptor for degradation ${ }^{118}$. Truncation mutations thus disrupt receptor trafficking and result in delayed receptor internalization and/or degradation ${ }^{115,117}$, increased cell surface expression of truncated CSF3R, sustained STAT5 activity, and enhanced cell proliferation ${ }^{119}$.

The capacity of the most common CSF3R mutation, CSF3RT618I, to drive leukemogenesis in CNL was established by Fleishman et al. using a murine bone marrow transplant model ${ }^{120}$. The CSF3RT618 mutation induced a lethal myeloproliferative disorder which recapitulated the clinical features of $\mathrm{CNL}$, supporting the role of CSF3RT618I as an oncogenic driver mutation and providing "proof of concept" to Maxson et al.'s seminal report ${ }^{120}$. Moreover, the CSF3RT618I mutation was confirmed to act via the JAK-STAT signaling pathway as the associated pathogenic granulocytosis and splenomegaly were sensitive to treatment with JAK inhibitor Ruxolitinib $^{120}$. An analogous study demonstrated the leukemogenic potential and likewise favorable response to Ruxolitinib of one of the alternate transmembrane domain mutations, CSF $3 R$ T640N, which behaves similarly to CSF3RT618I $\mathrm{I}^{112}$.

The proportions of $\mathrm{CNL} / \mathrm{aCML}$ patients harboring membrane-proximal only versus membrane-proximal concurrent with a truncating CSF3R mutation (compound mutant) are $75 \%$ and $25 \%$, respectively ${ }^{121}$. In their consideration of the scarcity of truncation-only CSF3R mutations in CNL, Maxson and Tyner raised the possibility that, contrary to membrane proximal mutations, truncation variants alone may be insufficient to drive leukemogenesis in $\mathrm{CNL}^{122}$. Consistent with this, truncation mutations display slower cell transformation kinetics in cellular assays and require ligand for activation of downstream signaling ${ }^{14}$. Finally, studies using knock-in mice expressing truncated CSF3R confirmed that these mutations in isolation were insufficient to induce leukemic transformation ${ }^{106}$. The biological basis for this attenuated pathogenicity of truncation mutations has recently been reasoned to be due to inefficient MAP kinase signaling ${ }^{123}$, providing a mechanistic explanation as to why CSF3R truncation mutations are usually found concurrently with other mutations such as T618I or T615A in CNL ${ }^{14,121}$.

Importantly, in Maxson et al.'s landmark study, the two prototypical CSF3R mutations showed differential susceptibilities to different TKIs: membrane proximal mutations responded preferentially to the JAK inhibitor Ruxolitinib while truncation mutations displayed sensitivity to inhibition with SRC kinase inhibitor Dasatinib ${ }^{14}$. This key finding validated the concept of distinct 
downstream signaling dysregulation in CNL involving either JAK-STAT or SRC kinase pathways and provided a rationale for molecularly directed therapeutic targeting with TKI. This study also confirmed the low frequency of CSF3R mutations in AML (later reaffirmed by Beekman et al., finding a prevalence of CSF $3 R$ mutations of $<0.5 \%$ in a series of 1446 consecutive cases of de novo $\mathrm{AML}^{124}$ ), and their absence in B-cell and T-cell ALL as well as in reactive neutrophilia ${ }^{14,76}$, suggesting that the CSF3R mutation represents the defining molecular lesion in CNL.

A follow-up study by Pardanani et al. took the approach of dividing patients into broad groups according to strictly WHO-defined disease subsets ${ }^{76}$. Mutations in CSF3R were analyzed in 35 suspected cases of CNL, 12 of which were subsequently confirmed to have WHO-defined CNL. Significantly, CSF3R mutations were detected in $100 \%$ of WHO-defined CNL cases and none of the cases of WHOdefined aCML, MG-associated CNL, PMF or $\mathrm{CMML}^{76}$. CSF3RT618I mutations were the most prevalent, detected in 10 of $12 \mathrm{CNL}$ patients for a mutational frequency of $83 \%$, while the remaining two patients harbored alternative CSF3R mutations M696T and $\mathrm{I}^{2} 98 \mathrm{I}^{76}$. This study thus critically endorsed the CSF3RT618I mutation as a sensitive and specific molecular marker for WHO-defined $\mathrm{CNL}$ and the authors consequently recommended its incorporation into current diagnostic criteria ${ }^{76}$.

Subsequent studies have both corroborated the lower mutational frequency of CSF3R mutations in aCML, which overall ranges from $0-40 \%^{14,19,125}$, and confirmed the high prevalence of CSF3R mutations in WHO-defined CNL, identifying these mutations in $80-100 \%$ of patients as shown by Cui et al. and Ouyang et al. ${ }^{17,126}$. In this latter publication, seven of the eight patients carried the CSF3RT618I mutation (including two harboring compound mutations), while the remaining patient displayed a CSF3RP733T mutation. This study supported the occurrence of CSF3RT618I mutations exclusively in WHOdefined CNL and validated this mutation as a sensitive and specific diagnostic marker in $\mathrm{CNL}^{17}$. Interestingly, this study also found CSF3R-mutated patients to have higher hemoglobin levels compared to their wild-type counterparts, a phenotypic characterization that remains to be validated ${ }^{17}$. Though the majority of data supports CSF3R as a sensitive and specific marker of $\mathrm{CNL}$, variable mutational frequencies have been reported. Meggendorfer et al. found that only six of 14 CNL patients (43\%) harbored CSF3R mutations and additionally identified CSF3R mutations in two of 58 (3.4\%) aCML patients and two of 146 (1.4\%) CMML patients ${ }^{19}$. This discrepancy is likely due to small sample sizes, non-uniformity in adopting strict WHO-defined diagnostic criteria, and inconsistencies in morphological diagnosis.

\section{Compound mutants}

A substantial portion of CSF3R-mutated CNL cases (up to $30 \%$ ) express compound mutations constituting of both membrane-proximal and truncation mutations on the same allele ${ }^{121}$. Because it was unclear whether compound mutants were sufficient to induce leukemogenesis in $\mathrm{CNL}$, Rohrabaugh et al. recently performed an elegant study demonstrating that CSF3R compound mutations induced aggressive lethal leukemia in mice and that BaF3 cells expressing CSF $3 R$ compound mutations in vitro were resistant to both JAK and SRC inhibitors ${ }^{123}$. Furthermore, they proved that both proximal and compound CSF3R mutations relied on enhanced MAPK signaling for oncogenic transformation and effectively abrogated leukemia development in mice expressing either of these mutational variants by targeting Mek1/2 with Trametinib $^{123}$. These results not only highlight the potential role of enhanced MAP kinase signaling in certain CSF3Rmutated myeloid malignancies but further suggest that patients harboring compound mutations may exhibit resistance to JAK inhibitors in practice ${ }^{123}$.

\section{Additional mutations in CNL}

The majority of patients with CSF3R-mutated CNL simultaneously harbor mutations in SETBP1, ASXL1, or other genes ${ }^{19,76,126}$. Furthermore, $~ 10-20 \%$ of CNL cases are negative for CSF3RT618I and other membrane-proximal CSF3R mutations suggesting that additional genetic lesions contribute to leukemic phenotype. In parallel, the genomic landscape of CNL is becoming increasingly complex with the recognition of a multitude of other mutations occurring either in conjunction with, or independent of those in CSF3R. In a recent review of the genomics of CNL, Maxson and Tyner comprehensively organized additional genetic lesions in CNL into either mutations in SETBP1, signaling mutations such as $J A K 2$, spliceosome complex mutations such as SRSF2 and U2AF1, or mutations impacting epigenetics such as $A S X L 1$ and $T E T 2^{122}$. Mutations in genes such as CALR have also been described in $\mathrm{CNL}^{127}$. Below is an outline of the most prevalent mutations in addition to CSF3R in CNL and if relevant, their clinical implications.

\section{SETBP1 mutations}

SET is a negative regulator of the tumor suppressor protein phosphatase 2A (PP2A). Set binding protein 1 (SETBP1) protects SET from protease cleavage, thus inhibiting PP2A activity and driving proliferation signals. SETBP1 overexpression is associated with decreased survival in AML, suggesting a role for SETBP1 in leukemogenesis $^{128}$. SETBP1 mutations have been identified in nearly $25 \%$ of aCML patients and have been associated 
with higher white blood cell count at diagnosis and inferior survival; ${ }^{129}$ they have also been identified, to a lesser extent, in MDS/MPN unclassified $(10 \%)^{129}$, CMML $(14.5 \%)$, and AML $(<1 \%)^{130}$. The prevalence of SETBP1 mutations in CNL has been reported to be between 14\% and $\sim 56 \%{ }^{19,76,121,126,131}$ and these mutations are enriched in CNL patients harboring CSF3R mutations ${ }^{121}$. Meggendorfer et al., Piazza et al., Pardanani et al., Elliott et al., Cui et al., and Gotlib found SETBP1 mutational frequencies of $14 \%{ }^{19}, 25 \%{ }^{129}, 33 \%{ }^{76}, 38 \%{ }^{18}, 42.9 \%{ }^{126}$, and $56 \%{ }^{121}$, respectively, in their series of CNL patients. With respect to the high prevalence of co-occurring SETBP1 and CSF3R mutations in CNL, Pardanani et al. and Cui et al., respectively, found that $40 \%{ }^{76}$ and $75 \%{ }^{126}$ of CSF3RT618I-mutated CNL patients co-expressed mutations in SET binding protein. Elliott et al. demonstrated that all five (100\%) of $13 \mathrm{CNL}$ patients carrying a SETBP1 mutation co-expressed a CSF3RT618I mutation ${ }^{18}$. In a recent review of $16 \mathrm{WHO}$-defined CNL patients, all of whom harbored the CSF3RT618I mutation, SETBP1 mutations were identified in $63 \%(10 / 16)^{132}$. Conversely, Meggendorfer et al. found that, although not statistically significant, SETBP1 and CSF3R mutations appeared to be mutually exclusive in their cohort of CNL patients ( 0 of six $)^{19}$.

The prognostic relevance of concurrent SETBP1 and CSF3R mutations remains to be clarified, though some evidence suggests this may be a mutationally detrimental combination. Pardanani et al. revealed a trend toward reduced survival in their cohort of double CSF3R-SETBP1 mutants; however, the analysis was limited by a small number of cases and requires validation ${ }^{76}$. Elliott et al. observed that of the two of 14 CNL patients who developed blast phase disease, both carried CSF3RSETBP1 (but not ASXL1) mutations, suggesting a role for SETBP1 mutations in blastic transformation ${ }^{18}$. Furthermore, in a report of a patient harboring both CSF3RT618I and SETBP1D868N mutations, Lasho et al. described resistance to treatment with JAK1 and JAK2 inhibitors in vitro and lack of clinical response to Ruxolitinib in vivo ${ }^{133}$. Similarly, Ammatuna et al. affirmed unresponsiveness to Ruxolitinib in a patient with CSF3RSETBP1-mutated disease, though this individual had $\mathrm{aCML}^{134}$. In contrast, another case of CNL harboring both CSF3R and SETBP1 mutations treated with Ruxolitinib showed hematological, clinical, and molecular improvement with reductions in both mutational allele burdens-although this patient died approximately 9 months after initiating treatment ${ }^{135}$. Finally, a recent meta-analysis by Shou et al. demonstrated a lack of prognostic impact of SETBP1 mutations in CNL, although these mutations were significantly associated with poor prognosis in MDS and $\mathrm{CMML}^{136}$.

\section{Cell-signaling genes \\ JAK2V617F mutations}

There have been several reports of JAK2V617F mutations in individuals with suspected CNL bearing typical features of the disease and lacking those of PV, ET, and $\mathrm{PMF}^{76,77,90,137-146}$. This putative association, however, must be interpreted in light of the fact that the $J A K 2 \mathrm{~V} 617 \mathrm{~F}$ mutation was often the reason for reporting and that importantly, most of these provisional cases were not tested for oncogenic CSF3R mutations as these were not yet described. Using the newly available data on CSF3R mutational status and updated WHO definitions of CNL, JAK2V617F and CSF3RT618I mutations now appear to be mutually exclusive ${ }^{76}$. A more recent report of 14 cases of strictly WHO-defined, CSF3R-positive CNL showed an absence of JAK2V617F mutations in all patients tested ${ }^{18}$. Another series by Gotlib et al. showed one patient with concurrent CSF3RG683R and $J A K 2 \mathrm{~V} 617 \mathrm{~F}$ mutations but the clonality of this double mutant could not be verified due to limited material ${ }^{121}$.

Nonetheless, rare cases of WHO-defined CNL may harbor JAK2 mutations. In a report of 35 cases of clinically suspected CNL in whom JAK2V617F screening was performed, three individuals harbored JAK2V617F mutations but only one with truly WHO-defined CNL (but lacking the prototypical CSF3R mutation) ${ }^{76}$, supporting the apparent mutual exclusivity of $J A K 2$ and CSF3R mutations, yet allowing for JAK2 positivity in exceptional cases of CNL. Though the JAK2V617F mutation serves as a marker of myeloid neoplasm and effectively establishes clonality, its prognostic implications in CNL are unclear. There is some evidence for a favorable impact with reported cases surviving beyond 2 years (and one surviving beyond 8 years treated with Hydroxyurea alone $)^{141}$. However, other data have depicted JAK2-mutated CNL cases with a more aggressive evolution and a high risk of transformation to $\mathrm{AML}^{90,138,143}$, confounding the prognostic relevance of this marker. There is thus currently a lack of convincing data to support any diagnostic or prognostic role of JAK2 mutations in $\mathrm{CNL}$, other than in corroborating clonality.

\section{Spliceosome-associated genes SRSF2 and U2AF1}

Mutations in spliceosome complex components such as SRSF2 and U2AF1 have uncommonly been reported in CNL. Meggendorfer et al. found SRSF2 mutations to be present in CNL with a mutational frequency of $21 \%{ }^{19}$. In a CNL case in which mutational analysis was performed, Senin et al. demonstrated mutations in CSF3RT618I, SETBP1G870S and SRSF2P95H ${ }^{147}$. Further, Dao et al. evaluated 10 cases of CSF3RT618I-positive myeloid neoplasms including aCML, CNL, or MPN not otherwise specified and unexpectedly found frequent mutations in 
U2AF1 (four of 10 cases of chronic myeloid neoplasms, not defined further in this study) ${ }^{148}$, which had previously been observed in primary myelofibrosis, MDS, and $\mathrm{CMML}^{130}$. The cooperative role of these and other spliceosome mutations in the pathogenesis of CNL, as well as their prognostic implications remain to be determined.

\section{Epigenetic modifiers}

ASXL1

ASXL1 regulates histone modification and has been shown to be mutated with variable but, in certain cases, high frequency in CNL. Reported mutational frequencies of ASXL1 mutations in CNL range from $30 \%$ to $81 \%^{18,19,132,148}$. Pardanani et al. identified ASXL1 mutations in the majority of CSF3R-mutated CNL patients (unpublished) ${ }^{131}$. The most common ASXL1 mutations observed are frameshift and non-sense mutations in exon 12 resulting in truncation of the gene product. In a critical study by Elliott et al., the presence of ASXL1 mutations (along with thrombocytopenia) was found to be independently predictive of shortened survival on multivariate analysis ${ }^{18}$. Furthermore, evolution of CNL to CMML was observed in patients harboring ASXL1 mutations and lacking SETBP1 suggesting a pathogenic role for $A S X L 1$ mutations in transformation to $\mathrm{CMML}^{18}$.

\section{TET2 mutations}

The TET proteins are dioxygenases that play a role in DNA methylation and somatic mutations in TET2 are frequently present in myeloid and, to a lesser extent, lymphoid malignancies ${ }^{149}$. In their cohort of $14 \mathrm{CNL}$ patients, Meggendorfer et al. found that close to $30 \%$ of them were TET2-mutated ${ }^{19}$. Interestingly, concomitant SRSF2 and TET2 mutations were not observed in any case, though this was not statistically significant ${ }^{19}$. In a recent report by Dao et al., a CNL patient negative for the CSF3R mutation but still meeting strict WHO-defined criteria for CNL harbored a TET2 mutation along with potentially pathogenic mutations in PTPN11 (a nonreceptor tyrosine kinase), TP53, DNMT3A, NF1, BCOR, BCORL1, PHF6, and RUNX1, as revealed by NGS ${ }^{150}$. Sporadic mutations in other epigenetic modifiers such as $E Z H 2$ and KDM6A have also been reported in $\mathrm{CNL}^{148}$.

In recent years, the involvement of TET2 and ASXL1 mutations in clonal hematopoiesis of indeterminate potential (CHIP) $)^{151}$ and the current observation that such mutations frequently co-occur with CSF3R mutations in CNL (sometimes with mutational frequencies up to $81 \%$ as is the case for $A S X L 1$ ) has led to the theory that CHIP may provide a backdrop for a later-occurring CSF3R mutation which, in turn, imparts a neutrophilic phenotype resulting in $\mathrm{CNL}^{122}$. In their recent review on $\mathrm{CNL}$ genomics, Maxson et al. provide three distinct models for mutation acquisition in $\mathrm{CNL}$, each with their own diagnostic, therapeutic and prognostic implications, with CSF3R mutations occurring as either a primary or secondary event ${ }^{122}$. This is a fascinating potential association and more data will be required in order to fully elaborate its mechanistic basis.

\section{Calreticulin}

Mutations in calreticulin are present in the majority of $J A K 2 / M P L$-negative cases of ET and $\mathrm{PMF}^{152,153}$. Lasho et al. provided the first account of CALR mutations in CNL in 2014 with their observation of a CNL patient harboring CSF3RT618I, SETBP1G870D, and a novel CALR point mutation (CALRE398D c.1194 G $>\mathrm{T})^{127}$. This variant was distinct from the classical CALR mutations found in ET or PMF, suggesting an alternate pathogenetic mechanism. Another series of CNL patients in the Mayo Clinic cohort also included one patient carrying the same distinct CALR mutation (E398D) ${ }^{18}$. Cui et al., in their study of 14 WHO-defined CNL patients found eight expressing the CSF3RT618I mutation, including one with a concurrent CALR exon 9 frame-shift mutation (c.1154-1155insTTGTC) ${ }^{126}$. Thus far, there are no clear prognostic or therapeutic implications of CALR mutations in $\mathrm{CNL}$, although the CALR mutant may become an interesting and relevant therapeutic target in the future.

\section{Additional mutations}

By using exome and RNA sequencing, Menezes et al. sought to identify candidate pathogenic genes in CNL and demonstrated an array of genomic lesions ${ }^{154}$. In addition to the classical CSF3RT618I mutation, mutations in genes involved in splicing machinery (LUC7L2 and U2AF1), protein kinases (PIM3-SCO2 fusion gene), and epigenetic regulation (TET2 and $A S X L 1)$ were identified in an index CNL patient.

The PIM3 oncogene belongs to the Serine-Threonine protein kinase family (PIM subfamily) and plays a role in regulating signal-transduction cascades driving cell proliferation and survival ${ }^{154}$. Mutations in PIM3 have been found to be enriched in hematological and epithelial tumors ${ }^{154}$. These results raised an interest for "cooperating mutations" and provided a rationale that these additional mutations may serve as new therapeutic targets for agents such as PIM kinase inhibitors. Though LUC7L2 mutations have previously been described in MDS, they have rarely been identified in $\mathrm{CNL}$ and their putative pathogenic impact remains uncertain ${ }^{155}$.

More recently, Langabeer et al. reported the results of NGS studies on a modest but informative cohort of four CSF3RT618I-mutated CNL patients, identifying additional mutations in all those tested: $\operatorname{SRSF2}(n=4 / 4)$, $\operatorname{SETBP} 1(n=3 / 4), \operatorname{NRAS}(n=1)$, and CBL $(n=1)^{156}$. 
Table 1 World Health Organization 2016 Revised Diagnostic Criteria for chronic neutrophilic leukemia ${ }^{a}$

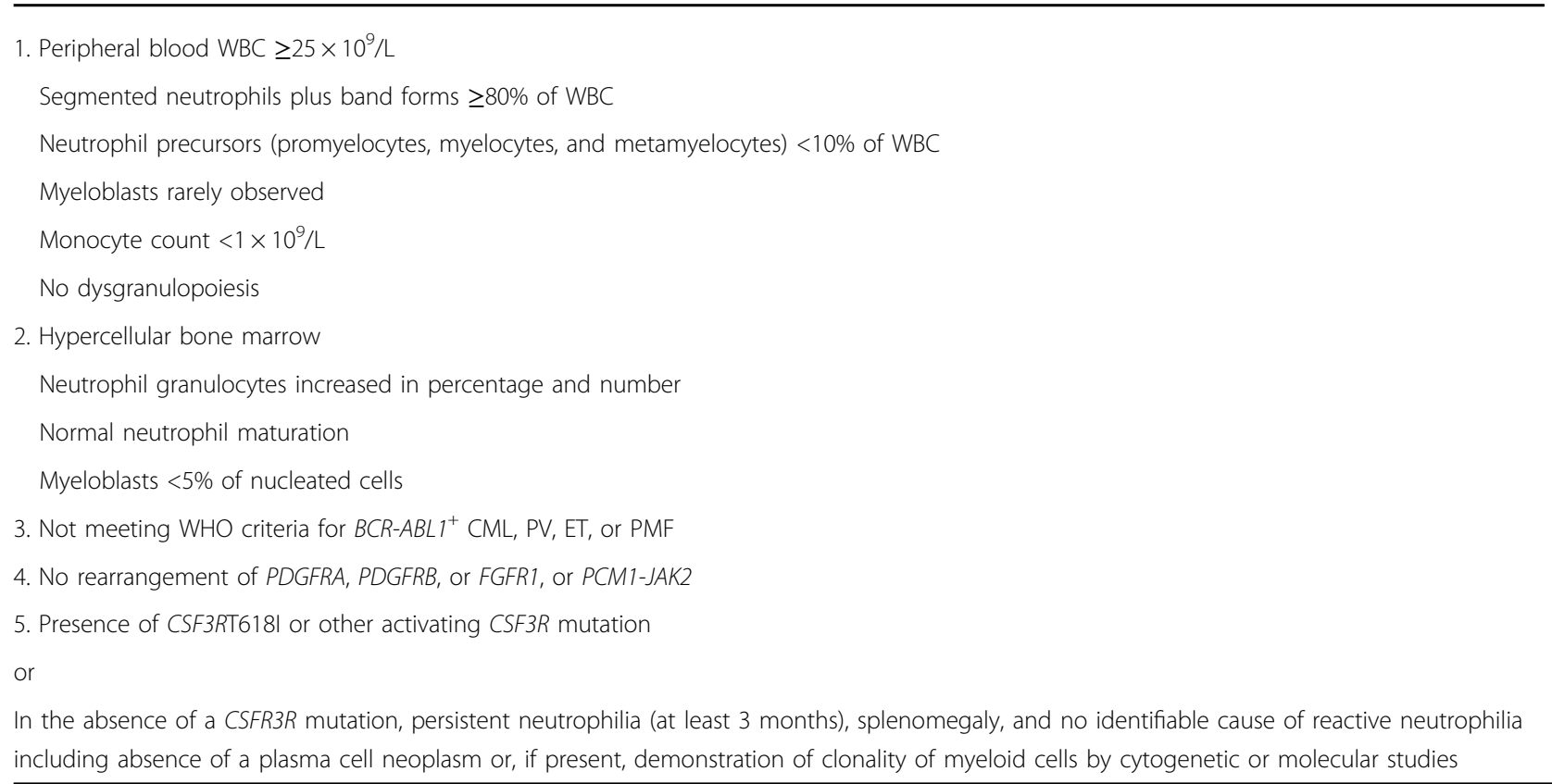

WBC white blood cells, CML chronic myeloid leukemia, PV polycythemia vera, ET essential thrombocythemia, $P M F$ primary myelofibrosis

a adapted from Arber et al. ${ }^{38}$

\section{Clonal evolution}

The acquisition of additional molecular or cytogenetic abnormalities during clonal evolution has been reported in $\mathrm{CNL}^{18,156}$. The abovementioned study by Langabeer et al. using NGS also included analyses at the time of blastic transformation which identified clonal evolution in all patients as evidenced by increasing CSF3RT618I allele frequency or gain/loss of mutations ${ }^{156}$. The finding of NRAS mutations both at diagnosis and during blast crisis in this study provides a rationale for use of MEK inhibitors in $\mathrm{CNL}^{156}$. In the CNL patient having transformed to blastic phase in a study by Elliott et al., new karyotypic aberrations in the form of acquisition of monosomy 5 and 7 were also noted ${ }^{18}$. Another report additionally noted trisomy 21 , deletion $12 \mathrm{p}$, and monosomy 7 at the time of blast transformation in three of $12 \mathrm{CNL}$ patients who were cytogenetically normal at baseline, though treatment with Hydroxyurea was postulated to have contributed to these karyotypic changes ${ }^{12}$. Recently, Nooruddin et al. provided valuable insight into the clonal evolution of CNL as acquisition of de novo mutations in KIT and GATA2 as well as increase in $R U N X 1$ allele frequency were present in an index CNL patient at the time of disease progression and are posited to have driven clonal evolution ${ }^{135}$.

\section{Mutation order}

The order of acquisition of CSF3R mutations relative to additional mutations in $S E T B P 1$, epigenetic modifiers, or spliceosome machinery has been determined only in isolated case reports and further studies are required in order to understand the impact of mutation chronology on the clonal evolution and progression of CNL.

\section{Revised 2016 WHO diagnostic criteria}

In 2016, the WHO classification of hematologic malignancies was updated to reflect the changes in the genomic landscape of CNL, endorsing the CSF3R mutation's "strong association" with $\mathrm{CNL}^{38}$. This justly represented the most significant modification with respect to the 2008 criteria. The presence of CSF3RT618I or another activating CSF3R mutation now comprises one of the five key diagnostic components of CNL. While these diseasedefining mutations serve as diagnostic markers if present, the WHO also allows for the possibility of CSF3R-negative disease in the face of chronic neutrophilia, splenomegaly, and the exclusion of reactive leukocytosis ${ }^{38}$. Though the absence of CSF3R mutation does not exclude the possibility of $\mathrm{CNL}$, however, it should prompt careful review of the diagnosis and consideration of alternative diagnoses.

The rest of the updated 2016 WHO diagnostic criteria for CNL remain relatively unchanged and are summarized in Table 1. The criteria include: peripheral blood leukocytosis of $\geq 25 \times 10^{9} / \mathrm{L}$ (of which $>80 \%$ are segmented neutrophils plus band forms and $<10 \%$ are neutrophil precursors with rare myeloblasts), monocyte count $<1 \times$ $10^{9} / \mathrm{L}$, and absence of dysgranulopoiesis, as well as hypercellular bone marrow with granulocyte hyperplasia, 
normal maturation, and $<5 \%$ myeloblasts ${ }^{38}$. The remaining components are exclusionary and consist of absence of WHO criteria for another MPN and absence of rearrangements in PDGFRA, PDGFRB, FGRF1, or the newly added $P C M 1-J A K 2^{38}$. If there is an underlying plasma cell disorder, myeloid clonality must be demonstrated in order to make the additional diagnosis of CNL.

\section{Prognosis and natural history}

Though the clinical course of $\mathrm{CNL}$ is variable, the overall prognosis is generally reserved with a substantial portion of patients eventually progressing to blast crisis. CNL has been historically described as having a "fatal clinical course"11. Overall median survival has been reported between 21 and 30 months in earlier reports, with a 5-year survival of $28 \%$ in a 2002 review by Reilly $^{6,157}$. A more recent series of 16 CNL patients, all of whom harbored the CSF3RT618I mutation, demonstrated a median overall survival of 24 months ${ }^{132}$ which is similar to the median overall survival reported from $40 \mathrm{CNL}$ cases by Elliott et al. (23.5 months) ${ }^{89}$. Unfortunately recent survival data ominously mirrors that of historical cohorts, showing little to no improvement over time. The transformation rate to acute myeloid leukemia (AML) has been shown to vary between $10 \%$ and $21.2 \%^{6,16}$ with a median time to AML transformation of 21 months (3-94 months) ${ }^{89,158}$.

The spectrum of fatal complications in CNL includes hemorrhagic diathesis, with fatal intracranial hemorrhage being particularly common in earlier reports ${ }^{11}$, progressive disease, blastic or leukemic transformation, and treatment-related toxicity following chemotherapy induction or transplantation ${ }^{12,158}$.

Distinct disease phases analogous to the accelerated and blastic phases observed in CML have not formally been defined in CNL, though its natural history often does recapitulate that of untreated CML. Disease progression in CNL typically involves resistance to treatment, progressive refractory neutrophilia, increasing red cell and platelet transfusion dependency, worsening organomegaly consistent with disease acceleration, and eventual blast crisis which to date, has been exclusively reported as myeloid. As discussed, such progression may be associated with the acquisition of additional cytogenetic abnormalities ${ }^{148}$.

Further, as in other classic MPN, there appears to be the potential to evolve toward/from other MPNs such as $\mathrm{PV}^{50,51}$ and $\mathrm{CMML}^{18}$.

\section{Prognostic markers}

There is currently no prognostic scoring system for $\mathrm{CNL}$ and due to the rarity of the disease, appropriately validating one would be anticipated to be challenging. Although it is tempting to extrapolate from other myeloid malignancies in assuming that factors such as advanced age, high blast count, extent of splenomegaly, and severity of cytopenias confer a negative prognosis, at this time there are no data to formally support this in CNL. Nevertheless, certain phenotypic and molecular features including leukocyte and platelet count as well as specific mutations and mutational combinations have emerged as potential prognostic markers in CNL.

Cui et al. recently studied 16 WHO-defined CNL patients and found prognostic relevance of an elevated WBC count above $50 \times 10^{9} / \mathrm{L}^{132}$. In this series, those presenting with $\mathrm{WBC}$ above $50 \times 10^{9} / \mathrm{L}$ had a median overall survival of 11 months compared with 39 months in those with $\mathrm{WBC}$ below $50 \times 10^{9} / \mathrm{L}^{132}$.

In a critical and comprehensive study, Elliott et al. evaluated the prognostic relevance of a number of factors including age, LDH, cytopenias, splenomegaly, bilirubin levels, and genetic variables such as SETBP1, ASXL1 and CSF3R mutations in 14 CSF3R-mutated CNL cases ${ }^{18}$. In univariate analysis, survival was detrimentally impacted by the presence $A S X L 1$ mutations, thrombocytopenia, and increased total bilirubin. However, on multivariable analysis, only the presence of ASXL1 mutations and thrombocytopenia remained independently adverse prognostic factors of decreased survival ${ }^{18}$. Borderline significance was found for both gender and elevated alkaline phosphatase in this study. Of interest, four (29\%) patients were alive after a median follow-up of 77.6 months, none of whom harbored ASXL1 mutations. The authors concluded that cooperative ASXL1 mutations were frequent (57\%) and independently predictive of a shortened overall survival in in CSF3R-mutated CNL patients, consistent with their negative prognostic role in other myeloid malignancies.

Interestingly, though they may have a pathogenic role in disease transformation and have been shown to confer refractoriness to treatment and a trend toward decreased survival $^{76,133}$, SETBP1 mutations were not an independent predictor of overall survival in the aforementioned study $^{18}$. A recent meta-analysis by Shou et al. also examined the prognostic significance of SETBP1 mutations in various myeloid malignancies including $\mathrm{CNL}^{136}$. Although they found that MDS and CMML patients exhibiting SETBP1 mutations had significantly worse prognosis than their wild-type counterparts, there wassimilar to Elliott et al.'s findings ${ }^{18}$ - no significant effect of SETBP1 mutations on prognosis in CNL, though both these studies were limited by relatively small sample sizes $^{136}$.

Screening for ASXL1 and SETBP1 mutations in both CSF3R-mutated and unmutated CNL patients could thus be advocated in light of their potential prognostic significance (for ASXL1) and their value in ascertaining clonality. 


\section{Management}

Effective management translating to a survival benefit in $\mathrm{CNL}$ has been hampered by the rarity of the disease, the lack of prospective trials, and the limited therapeutic options, which have themselves, largely been borrowed from the arsenal of therapies utilized in other MPN. In 2002, Bohm et al. expressed that "because of the rarity of the disease, no therapeutic standard has been determined"11 and this remains true to this day as there still is no current standard of care in CNL. Aside from hematopoietic stem cell transplant (HSCT), which itself is restricted to a minor portion of CNL patients, none of the commonly used therapeutic agents provide diseasemodifying effects and no significant therapeutic advances have been made to improve this.

Both splenic irradiation and splenectomy have been used since 1979 for the palliation of symptomatic splenomegaly in $\mathrm{CNL} ;{ }^{7}$ and interestingly, in fact, the index case reported by Tuohy was treated with splenectomy ${ }^{1}$. However, due to reports of worsening neutrophilia postoperatively, splenectomy cannot be recommended as part of the treatment approach for $\mathrm{CNL}^{1,10}$.

Reports of use of oral chemotherapy in CNL also date back to the late 1970's and early 1980's ${ }^{7,10,159}$. Classically, agents such as Busulfan and Hydroxyurea have been used to temporarily control leukocytosis. Although they may help achieve disease stability for a period of time, however, they inevitably lose their efficacy and offer no curative potential.

Hydroxyurea has historically been the most frequently utilized agent with up to $75 \%$ of patients showing an initial response as assessed by reduced leukocytosis and/ or splenomegaly with median duration of therapy of 12 months (range: 6-87) ${ }^{12}$. Surprisingly, in this study, two patients continued in the chronic stable phase on Hydroxyurea for 18 and 87 months, respectively ${ }^{12}$. In a 2015 report from the Mayo Clinic of 14 cases of CNL, Hydroxyurea was the most frequently used therapy and was ultimately received by all patients in this series ${ }^{18}$. Despite initially controlling hematological parameters, most patients became refractory, eventually displaying increased transfusion dependence. Furthermore, up to $\sim 25 \%$ of cases have been reported to be refractory to such therapy from the outset ${ }^{12}$.

Other therapies in the 2015 Mayo series included Interferon, hypomethylating agents, Ruxolitinib, Thalidomide, Cladribine, and Imatinib ${ }^{18}$ and many other accounts of use of these agents have been published, as they constitute the most common therapeutic approach $^{6,11,12}$. Other therapies that have been attempted historically include low-dose Cytarabine, 6-Thioguanine (6-TG), 2-Chlorodeoxyadenosine, and leukapheresistypically with short-lived responses ${ }^{12}$. These agents thus provide a palliative and usually transient benefit and all fail to induce remission.

Treatment with some of these agents, including Cladribine, Thalidomide, and Ruxolitinib have also been attempted following refractoriness to Hydroxyurea with, again, typically short-lived responses ${ }^{12,18}$.

\section{Interferon}

Interferon-alpha (IFN-a) has a long history of use in $\mathrm{CNL}$ and is the only therapeutic agent to offer the potential for durable remissions as published in limited case reports $^{11,18,140,157 .}$

Meyer et al. reported two patients with characteristic features of $\mathrm{CNL}$ treated with IFN-a ${ }^{157}$. At initiation of therapy, both patients had progressive disease, including one who was refractory to Hydroxyurea treatment. Both patients achieved long-term stabilization of their disease with IFN therapy duration of 16 and 26 months, respectively. The first patient showed slow progression, though still did not require therapy, after discontinuing IFN (follow-up period 90 months), and the second patient was stable receiving ongoing therapy (follow-up period 66 months). In one of the patients, the dose of IFN could be significantly reduced during maintenance without relapse. Moreover, neither presented infectious or hemorrhagic complications under therapy. This study demonstrated that IFN-a was not only safe and effective in CNL (even in pretreated and actively progressing patients), but that it also could induce durable remissions ${ }^{157}$. A review of 14 cases of CNL by Bohm et al. included a patient treated with IFN-a who maintained a clinical remission for the duration of 41 months, corroborating Meyer's findings ${ }^{11}$. Similarly, Zhang et al. described a JAK2V617F-mutated CNL patient treated with IFN-a whose WBC counts were controlled below $10 \times 10^{9} / \mathrm{L}$ and who remained clinically well during maintenance IFN treatment (though duration of remission was not specified ${ }^{140}$. Yet another retrospective series included one patient treated with IFN (along with Hydroxyurea co-treatment) who attained prolonged partial response for at least 2 years, after which IFN was discontinued due to disease progression ${ }^{18}$.

These limited studies must evidently be counterbalanced with the fact that not all patients will respond to or tolerate treatment with interferon ${ }^{160}$, though they do provide a rationale for continuing to include this agent in our therapeutic arsenal for CNL.

\section{Induction chemotherapy}

Unfortunately, despite the aggressivity of induction chemotherapy, it has not been reported to induce hematological remission in CNL. There is one account of a patient in blast phase attaining a second chronic phase following AML induction-type " $7+3$ " chemotherapy ${ }^{10}$; 
however, the majority of patients are either refractory or succumb to treatment-related mortality, most notably complications of cytopenias including intracranial hemorrhage and sepsis ${ }^{11,12,89}$.

\section{Hematopoietic stem cell transplant}

Though the aforementioned therapeutic agents may induce temporary disease stability or, in rare instances, durable remissions as may be the case with IFN, none have yielded disease-modifying effects. As most described cases of CNL occur in elderly patients, with only three cases under the age of 40 years being reported as of 1996, accounts of allogeneic HSCT were conceivably rare in early literature. The first report of HSCT in CNL was in 1996 by Hasle et al. describing two young individuals ( 15 and 25 years old) treated with allogeneic $\mathrm{HSCT}^{10}$. Both were in remission 6.5 and 4.5 years post-HSCT, respectively. Other earlier publications by Bohm et al. and Piliotis et al. reported cases of young patients with remissions of up to 73 months after related allogeneic $\mathrm{HSCT}^{11,88}$. Likewise, Goto described the case of a young CNL patient transplanted in stable phase, this time using an unrelated HLA matched donor, with remission persisting beyond 3 years ${ }^{161}$. The majority of reported cases of HSCT in CNL thus far have consisted of sibling donors, though at least two reports including that of Goto, relay cases of unrelated donors ${ }^{138,161}$.

Transplant has been described in all phases of disease. Notably, patients having received transplant in blast phase appear to have experienced worse outcomes with regards to regimen-related toxicity and/or early relapse ${ }^{12,138}$. Kako et al. reported a case of a 46-year-old male with $J A K 2$ V617F-positive CNL who underwent allogeneic HSCT in progressive phase and developed early systemic relapse and central nervous system infiltration ${ }^{138}$. In a review of transplant outcomes in $\mathrm{CNL}$, it was demonstrated that $71 \%$ of patients who received HSCT in chronic phase had an ongoing remission lasting over 7 months compared to shorter remission durations for those transplanted in accelerated phase ${ }^{158}$, altogether suggesting that a more favorable outcome may be achieved when transplantation is undertaken during the chronic phase of disease.

The presence of the CSF3R mutation may potentially serve as a tool for monitoring patients post-transplant when present at baseline. Lee et al. described patients with both CNL and CMML harboring CSF3RT618I mutations having undergone allogeneic $\mathrm{HSCT}^{162}$. In both cases, the CSF3RT618I mutation was not detected following allogeneic HSCT as the patients remained in remission, suggesting a predictive role of this mutation in posttransplant relapse ${ }^{162}$. Prior studies showing a correlation between post-transplant relapse and the persistence of the
CSF3RT618I in aCML support this and endorse monitoring of CSF3R mutant allele burden as a marker of transplant efficacy ${ }^{163}$.

Given the limited efficacy of available therapeutic agents -and despite the rare accounts of remissions with less toxic regimens-it is currently recommended that eligible patients be directed toward HSCT. Because the principle of "risk-adapted therapy" does not formally apply to CNL as it does to other MPN, the precept of "transplant if you can" is probably a safe approach in managing this aggressive and often rapidly fatal disease. Though optimal timing of transplant is uncertain, there is some data to preferentially support transplant in chronic phase ${ }^{12,138,158}$. Consequently, it may be advisable to have eligible patients evaluated for transplant early in their disease course, prior to blast transformation. The scarcity of data on outcomes following transplant, particularly when using alternative stem cell sources, such as cord blood, or nonmyeloablative approaches constitutes one of the many challenges in CNL and will require a collective effort in order to achieve progress on this front.

\section{JAK inhibitors}

The JAK1/2 inhibitor Ruxolitinib is currently approved for treatment of patients with intermediate- or high-risk myelofibrosis and, more recently, for PV patients intolerant or refractory to Hydroxyurea ${ }^{164,165}$. Due to the pivotal role of the JAK-STAT pathway in oncogenic signaling in $\mathrm{CNL}$, it was reasoned that Ruxolitinib may provide clinical efficacy in this disease. Below we provide a summary of studies evaluating Ruxolitinib in both murine models and humans.

The original study by Maxson et al. included a patient harboring the CSF3RT618I mutation who was treated with Ruxolitinib $10 \mathrm{mg}$ orally twice daily ${ }^{14}$. The patients' cells exhibited sensitivity to Ruxolitinib in vitro and in vivo and clinical effects included a significant reduction in white blood cell and absolute neutrophil count as well as a normalization of platelet count. Further blood count decreases occurred with escalation of the dose to $15 \mathrm{mg}$ orally twice daily. What was initially reported as a 5month response translated into a longer-term one as the patient was shown to still be responding after 11 months of therapy ${ }^{121}$. The "proof of concept" study by Fleishman et al. also showed a salutary effect of Ruxolitinib in a mouse model transplanted with CSF3RT618I-expressing hematopoietic cells ${ }^{120}$. Use of Ruxolitinib in this model suppressed CSF3RT618I-induced cell proliferation, controlled leukocytosis, reduced spleen weight, and increased mouse body weight, mirroring the symptomatic improvements seen in humans ${ }^{120}$. In contrast, Elliott et al. reported on a CNL patient with a more mitigated response to Ruxolitinib as the individual eventually 
required addition of Hydroxyurea in order to achieve disease control ${ }^{18}$.

Three studies evaluated the role of Ruxolitinib in the treatment of CNL patients co-expressing CSF3R and SETBP1 mutations with varying results ${ }^{133,135,166}$. Lasho et al. described a case of WHO-defined CNL with concurrent CSF3RT618I and SETBP1 mutations failing treatment with Hydroxyurea subsequently treated with Ruxolitinib $10 \mathrm{mg}$ orally daily in addition to Hydroxyurea $^{133}$. The patient became refractory to treatment with both agents, displaying worsening leukocytosis despite Ruxolitinib $20 \mathrm{mg}$ orally twice daily and Hydroxyurea $1 \mathrm{~g}$ orally daily. It is notable that Ammatuna described the same refractoriness to treatment in a CSF3RT618I and SETBP1 double mutant, but in WHOdefined $\mathrm{aCML}^{134}$. This discrepant lack of efficacy compared to the above-mentioned studies raised the possibility that co-expression of CSF3RT618I and SETBP1 may hamper JAK inhibitor therapy. Counter to this concept however, both Nooruddin et al. and Stahl et al. reported cases of CNL with concurrent CSF3RT618I and SETBP1G870S mutations initiated on Ruxolitinib titrated up to $20 \mathrm{mg}$ orally daily with periods of response lasting from 5 to 9 months ${ }^{135,166}$. In the former study, the patient noted symptom improvement after only 2 weeks of treatment and in the latter case, the additional presence of the ASXL1 mutation, known to confer a worse prognosis in CNL, may have biased the study ${ }^{166}$.

Similarly, it was found that harboring compound CSF3R mutations, as opposed to isolated proximal or truncation mutations, conferred resistance to Ruxolitinib in a murine model of CNL, suggesting that JAK inhibitors may be of minimal benefit to the $\sim 1 / 3$ of CNL patients exhibiting such compound CSF3R mutations ${ }^{123}$.

The question of whether JAK inhibitor therapy could achieve reduction in CSF3R allele burden was initially addressed by Dao et al. in an aCML patient harboring a CSF3RT618I (along with CBLI383T and KDM6AS114C) mutation $^{167}$. Treatment with Ruxolitinib resulted in hematological and symptomatic improvement, although no impact on allele burden was observed, suggesting the unlikeliness of Ruxolitinib having a selective anti-clonal or disease-modifying effect ${ }^{167}$. More recently, however, data have emerged demonstrating reduction in CSF3R allele burden consequent to Ruxolitinib therapy. Nooruddin et al. observed a parallel decrease in CSF3R and SETBP1 mutation allele burden in response to Ruxolitinib therapy $^{135}$. In this case, the acquisition of de novo mutations in KIT and GATA2 as well as increase in RUNX1 allele frequency are postulated to have played a role in disease evolution and/or Ruxolitinib resistance, but this requires validation ${ }^{135}$. Furthermore, Gunawan et al. reported on a patient with CNL harboring compound CSF3R mutations (CSF3RT618I and CSF3RG739 truncation mutation) in whom treatment with Ruxolitinib induced hematological response in parallel with reductions in allelic burden for both mutations ${ }^{168}$. Molecular relapse coincided with hematological relapse at day $784^{168}$. It is noteworthy that despite initial decline in CSF3R allele burden, this patient did not show symptomatic improvement, raising the question of whether reduction in CSF3R allele burden translates to a clinically meaningful benefit.

The host of variables, molecular and otherwise, underlying differential responses and eventual refractoriness to Ruxolitinib remains to be formally elucidated. It has been speculated that mechanisms involved in Ruxolitinib resistance in CNL may model those already described by Koppikar et al., namely heterodimeric reactivation of JAKSTAT by other JAK kinases ${ }^{169}$. It is also plausible that an individual's response (or lack thereof) to treatment with JAK inhibitor therapy is in part molecularly-driven and it will be important to determine how additional mutations such as those in ASXL1, SETBP1, or SRSF2 modulate the clinical effect of Ruxolitinib. Correspondingly, the attenuated sensitivity of compound CSF3R mutations to Ruxolitinib in certain studies ${ }^{123}$ suggests that additional aberrant signaling pathways are involved, possibly via activation of MAPK downstream signaling by alternative cellular signaling networks.

A prospective multicenter phase II clinical trial evaluating safety and efficacy of Ruxolitinib in CNL patients is currently underway (NCT02092324) and is expected to clarify Ruxolitinib's role in the management algorithm of $\mathrm{CNL}$. At the present time, considering the dearth of therapeutic options and some encouraging in vivo data, it would be reasonable to consider Ruxolitinib in the therapeutic arsenal for HSCT-ineligible CNL patients ${ }^{131}$.

\section{Novel therapeutic targets}

Apart from the aforementioned study involving Ruxolitinib, there are, at this time, no other investigational agents undergoing study for use in CNL. The finding of NRAS mutations in a portion of CNL patients has led to the rationale that treatment with MEK inhibitors such as Trametinib may offer disease control in certain mutationally-defined subsets, as it has been shown to do in other myeloid malignancies including $\mathrm{aCML}^{170,171}$. Rohrabaugh et al. supported this concept, showing that in vivo inhibition of MEK $1 / 2$ by Trametinib in a murine CNL model expressing compound CSF3R mutations effectively abrogated leukemia development ${ }^{123}$. These studies seemingly justify the evaluation of MEK1/2 inhibitors in patients with similarly molecularly annotated $\mathrm{CNL}$. As we gain more insight into the genomics and pathobiology of CNL, it is likely that molecularly driven targeted therapy will increasingly emerge as a compelling treatment approach. 


\section{Conclusion}

CNL remains a rare but often clinically aggressive MPN with few effective treatment options. Substantial progress has been made since the first description of the disease, most notably with the discovery of oncogenic CSF3R driver mutations in the majority of CNL patients. There now exists a more intricate molecular framework underpinning CNL biology than was originally thought. With the recent findings of additional genetic lesions in a substantial proportion of CNL patients, and considering the lack of disease-defining CSF3R mutations in others, one of the critical challenges will be molecularly anatomizing this disease: determining additional driver and ancillary mutations, their cooperativity, their interactions with cytogenetic and other extrinsic factors, and their phenotypic, therapeutic, and prognostic implicationsand ultimately integrating these variables into an operationally useful schema for prognostication and treatment. The order of mutation acquisition in CNL will also be important to define as it too may impact prognosis and response to therapy.

The new WHO diagnostic criteria reflect the strides in our knowledge of CNL pathogenesis as well as our increasingly sophisticated and available molecular diagnostic tools. Molecularly annotated sub-categories of $\mathrm{CNL}$ are emerging, contingent upon whether patients are CSF3R-negative, CSF3R-positive (proximal or truncation), compound-mutated, or carriers of CSF3R plus additional genetic lesions, potentially each with their own phenotypic and prognostic relevance. Molecular testing and diagnostic criteria will inevitably evolve in parallel to become more profoundly individualized, justly reflecting the genomic complexity of the disease.

As the collective cohort of CNL patients grows, along with our experience, we hope to achieve an even deeper understanding of the molecular, cytogenetic, and extrinsic factors driving disease initiation, transformation, and response to treatment. The optimal timing of transplant remains to be defined, just as the role of Ruxolitinib needs to be circumscribed in the therapeutic algorithm for CNL. Furthermore, new potentially relevant molecular targets have been identified and should be explored in order to maximize therapeutic alternatives. Finally, it is imperative that these advances in knowledge-this new science-not only reshape our vision of CNL but hopefully translate to meaningful and practical improvements in patient management and outcomes.

\section{Conflict of interest}

The authors declare that they have no conflict of interest.

\section{Publisher's note}

Springer Nature remains neutral with regard to jurisdictional claims in published maps and institutional affiliations.
Received: 2 November 2017 Revised: 1 December 2017 Accepted: 11 December 2017

Published online: 13 February 2018

\section{References}

1. Tuohy, E. A case of splenomegaly with polymorphonuclear neutrophil hyperleukocytosis. Am. J. Med. Sci. 160, 18-25 (1920).

2. Emile-Weil, P. \& See, G. La leucémie myélogène à polynucléaires neutrophiles. Presse Méd. 40, 1071-1074 (1932).

3. Jackson, I. M. D. \& CR A case of neutrophilic leukemia. Am. J. Clin. Sci. 249, 72-74 (1965).

4. Tanzer, J., Harel, P., Boiron, M. \& Bernard, J. Cytochemical and cytogenetic findings in a case of chronic neutriphilic leukaemia of mature cell type. Lancet 1, 387-388 (1964).

5. Rubin, H. Chronic neutrophilic leukemia. Ann. Intern. Med. 65, 93-100 (1966).

6. Reilly, J. T. Chronic neutrophilic leukaemia: a distinct clinical entity? Br. J. Haematol. 116, 10-18 (2002).

7. You, W. \& Weisbrot, I. M. Chronic neutrophilic leukemia. Report of two cases and review of the literature. Am. J. Clin. Pathol. 72, 233-242 (1979).

8. Harris, N. L. et al. World Health Organization classification of neoplastic diseases of the hematopoietic and lymphoid tissues: report of the Clinical Advisory Committee meeting-Airlie House, Virginia, November 1997. J. Clin. Oncol. 17, 3835-3849 (1999).

9. Jaffe, E. S., Harris, N. L., Stein, H. \& Vardiman, J. W. World Health Organization Classification of Tumours: Pathology and Genetics of Tumours and Lymphoid Tissue. (IARC Press, Lyon, France, 2001).

10. Hasle, H., Olesen, G., Kerndrup, G., Philip, P. \& Jacobsen, N. Chronic neutrophil leukaemia in adolescence and young adulthood. Br. J. Haematol. 94, 628-630 (1996).

11. Bohm, J. \& Schaefer, H. E. Chronic neutrophilic leukaemia: 14 new cases of an uncommon myeloproliferative disease. J. Clin. Pathol. 55, 862-864 (2002).

12. Elliott, M. A. et al. WHO-defined chronic neutrophilic leukemia: a long-term analysis of 12 cases and a critical review of the literature. Leukemia 19, 313-317 (2005).

13. Bain, B. J. \& Ahmad, S. Chronic neutrophilic leukaemia and plasma cell-related neutrophilic leukaemoid reactions. Br. J. Haematol. 171, 400-410 (2015).

14. Maxson, J. E. et al. Oncogenic CSF3R mutations in chronic neutrophilic leukemia and atypical CML. N. Engl. J. Med. 368, 1781-1790 (2013).

15. SEER Hematopoietic and Lymphoid Neoplasm Database; Chronic Neutrophilic Leukemia. https://seer.cancer.gov/seertools/hemelymph/ 51f6cf58e3e27c3994bd5402/

16. Elliott, M. A. Chronic neutrophilic leukemia and chronic myelomonocytic leukemia: WHO defined. Best Pract. Res. Clin. Haematol. 19, 571-593 (2006).

17. Ouyang, Y., Qiao, C., Chen, Y. \& Zhang, S. J. Clinical significance of CSF3R, SRSF2 and SETBP1 mutations in chronic neutrophilic leukemia and chronic myelomonocytic leukemia. Oncotarget 8, 20834-20841 (2017).

18. Elliott, M. A. et al. ASXL1 mutations are frequent and prognostically detrimental in CSF3R-mutated chronic neutrophilic leukemia. Am. J. Hematol. 90, 653-656 (2015).

19. Meggendorfer, $M$. et al. Specific molecular mutation patterns delineate chronic neutrophilic leukemia, atypical chronic myeloid leukemia, and chronic myelomonocytic leukemia. Haematologica 99, e244-e246 (2014).

20. Nakamine, H. et al. Lymph node involvement in chronic neutrophilic leukemia. An immunohistochemical study. Virchows Arch. A Pathol. Anat. Histopathol. 412, 241-245 (1988).

21. Mitsumori, T., Komatsu, N. \& Kirito, K. A CSF3R T618l mutation in a patient with chronic neutrophilic leukemia and severe bleeding complications. Intern. Med. 55, 405-407 (2016).

22. Noguchi, T. et al. Severe bleeding tendency caused by leukemic infiltration and destruction of vascular walls in chronic neutrophilic leukemia. Int. J. Hematol. 74, 437-441 (2001).

23. Shigekiyo, T. et al. Bleeding tendency in chronic neutrophilic leukemia. Int. J. Hematol. 88, 240-242 (2008).

24. Hossfeld, D. K., Lokhorst, H. W. \& Garbrecht, M. Neutrophilic leukemia accompanied by hemorrhagic diathesis: report of two cases. Blut. 54, 109-113 (1987).

25. Ohtsuki, T. et al. Elevated neutrophil function in chronic neutrophilic leukemia. Am. J. Hematol. 41, 50-56 (1992).

26. Higuchi, T. et al. Transition of polycythemia vera to chronic neutrophilic leukemia. Leuk. Lymphoma 33, 203-206 (1999). 
27. Hirayama, Y. et al. [A case of chronic neutrophilic leukemia accompanied with severe bone marrow fibrosis which was effectively treated by hydroxyurea]. Rinsho Ketsueki 35, 1329-1334 (1994).

28. Bain B. J. B. R., Vardiman J. \& Thiele J. in WHO Classification of Tumours of Haematopoietic and Lymphoid Tissue: Chronic neutrophilic leukaemia. (eds Swerdlow, S. C. E. \& Harris, N. L.) (World Health Organization, Geneva, Switzerland, 2008).

29. Oogushi, K. et al. The discrepancy between chemotaxis and leukotriene B4 production in a patient with chronic neutrophilic leukemia. Jpn. J. Med. $\mathbf{2 8}$ 717-721 (1989)

30. Mehrotra, P. K., Winfield, D. A. \& Fergusson, L. H. Cellular abnormalities and reduced colony-forming cells in chronic neutrophilic leukaemia. Acta Haematol. 73, 47-50 (1985)

31. Silva, P. R. et al. Diagnosis, complications and management of chronic neutrophilic leukaemia: a case report. Oncol. Lett. 9, 2657-2660 (2015).

32. Hidalgo, C. et al. [Chronic neutrophilic leukemia: apropos of 2 cases and review of the literature]. Med. Clin. 95, 421-423 (1990).

33. Kojima, K. et al. Familial occurrence of chronic neutrophilic leukaemia. Br. J. Haematol. 105, 428-430 (1999).

34. Eichenhorn, M. S. \& Van Slyck, E. J. Marked mature neutrophilic leukocytosis: a leukemoid variant associated with malignancy. Am. J. Med. Sci. 284, 32-36 (1982).

35. Pane, F. et al. Neutrophilic-chronic myeloid leukemia: a distinct disease with a specific molecular marker (BCR/ABL with C3/A2 junction). Blood $\mathbf{8 8}$, 2410-2414 (1996).

36. Pane, F. et al. Neutrophilic-chronic myeloid leukemia: a distinct disease with a specific molecular marker (BCR/ABL with C3/A2 junction) [see comments]. Blood 88, 2410-2414 (1996).

37. Verstovsek, S. et al. Neutrophilic-chronic myeloid leukemia: low levels of p230 BCR/ABL mRNA and undetectable BCR/ABL protein may predict an indolent course. Cancer 94, 2416-2425 (2002).

38. Arber, D. A. et al. The 2016 revision to the World Health Organization classification of myeloid neoplasms and acute leukemia. Blood 127, 2391-2405 (2016)

39. Mizutani, Y., Okada, Y., Terachi, T., Kakehi, Y. \& Yoshida, O. Serum granulocyte colony-stimulating factor levels in patients with urinary bladder tumour and various urological malignancies. Br. J. Urol. 76, 580-586 (1995).

40. Kobashi, Y., Okimoto, N. \& Sakamoto, K. Squamous cell carcinoma of the lung producing granulocyte colony-stimulating factor and resembling a malignant pleural mesothelioma. Intern. Med. 43, 111-116 (2004).

41. Nishimura, M. et al. Autocrine growth by granulocyte colony-stimulating factor in malignant mesothelioma. Ann. Thorac. Surg. 82, 1904-1906 (2006).

42. Usami, N. et al. Granulocyte colony-stimulating factor-producing malignant pleural mesothelioma. J. Thorac. Oncol. 2, 257-258 (2007).

43. Yoshimoto, A., Kasahara, K., Saito, K., Fujimura, M. \& Nakao, S. Granulocyte colony-stimulating factor-producing malignant pleural mesothelioma with the expression of other cytokines. Int. J. Clin. Oncol. 10, 58-62 (2005).

44. Iwasa, K. et al. Anaplastic thyroid carcinoma producing the granulocyte colony stimulating factor (G-CSF): report of a case. Surg. Today 25, 158-160 (1995)

45. Jardin, F. et al. Intense paraneoplastic neutrophilic leukemoid reaction related to a G-CSF-secreting lung sarcoma. Am. J. Hematol. 80, 243-245 (2005).

46. Lou, E., Goodwin, J., Howell, D. N., Hicks, J. \& Brett Caram, L. A G-CSF-secreting adrenal carcinoma with rhabdoid-like differentiation causing leukocytosis. Nat. Rev. Urol. 6, 392-397 (2009)

47. Endo, K. et al. Gastric adenosquamous carcinoma producing granulocytecolony stimulating factor. Gastric Cancer 8, 173-177 (2005).

48. Omura, N., Abe, S., Hirai, K. \& Aoki, T. A case of granulocyte-colony stimulating factor producing gallbladder cancer. Am. J. Gastroenterol. 94, 273-275 (1999)

49. Schniewind, B. et al. Paraneoplastic leukemoid reaction and rapid progression in a patient with malignant melanoma: establishment of KT293, a novel G-CSF-secreting melanoma cell line. Cancer Biol. Ther. 4, 23-27 (2005).

50. Billio, A. et al. Chronic neutrophilic leukemia evolving from polycythemia vera with multiple chromosome rearrangements: a case report. Haematologica 86, 1225-1226 (2001)

51. Foa, P. et al. Chronic neutrophilic leukemia associated with polycythemiavera - pathogenetic implications and therapeutic approach. Br. J. Haematol. 78, 286-288 (1991)

52. Iurlo, A. et al. Polycythemia-vera terminating in chronic neutrophilic leukemia - report of a case. Am. J. Hematol. 35, 139 (1990).
53. Lee, S. S. et al. A case of transition of polycythemia vera to chronic neutrophilic leukemia. Korean J. Intern. Med. 19, 285-288 (2004).

54. Lugassy, G. \& Farhi, R. Chronic neutrophilic leukemia associated with polycythemia vera. Am. J. Hematol. 31, 300-301 (1989).

55. Harada, Y., Katano, T., Nakamura, Y. \& Adachi, Y. [A case of chronic neutrophilic leukemia associated with polycythemia vera]. Rinsho Ketsueki 34, 738-742 (1993)

56. Fujisawa, S. et al. [Polycythemia vera terminating in chronic neutrophilic leukemia: studies on in vitro growth of hematopoietic progenitor cells]. Rinsho Ketsueki 33, 1863-1868 (1992).

57. Watanabe, A. et al. A case of chronic neutrophilic leukemia with paraproteinemia (Igg type-lambda and Iga type-K). Jpn. J. Med. 23, 39-44 (1984).

58. Cehreli, C., Undar, B., Akkoc, N., Onvural, B. \& Altungoz, O. Coexistence of chronic neutrophilic leukemia with light chain myeloma. Acta Haematol. 91, 32-34 (1994).

59. Dincol, G. et al. Coexistence of chronic neutrophilic leukemia with multiple myeloma. Leuk. Lymphoma 43, 649-651 (2002).

60. Franchi, F., Seminara, P. \& Giunchi, G. Chronic neutrophilic leukemia and myeloma - report on long survival. Tumori 70, 105-107 (1984).

61. Lewis, M. J., Oelbaum, M. H., Coleman, M. \& Allen, S. An association between chronic neutrophilic leukemia and multiple-myeloma with a study of cobalamin-binding proteins. Br. J. Haematol. 63, 173-180 (1986).

62. Rovira, M., Cervantes, F., Nomdedeu, B. \& Rozman, C. Chronic neutrophilic leukemia preceding for 7 years the development of multiple-myeloma. Acta Haematol. 83, 94-95 (1990).

63. Standen, G. R., Jasani, B., Wagstaff, M. \& Wardrop, C. A. J. Chronic neutrophilic leukemia and multiple-myeloma - an association with lambda-light chain expression. Cancer 66, 162-166 (1990).

64. Troussard, $X$. et al. Neutrophilic leukemia and multiple-myeloma - 2 casereports. Ann. Med. Intern. 143, 136-139 (1992).

65. Zoumbos, N. C., Chrysanthopoulos, C., Starakis, J. \& Kapataiszoumbos, K Kappa-light chain myeloma developing in a patient with chronic neutrophilic leukemia. Br. J. Haematol. 65, 504-505 (1987)

66. Uppal, G. \& Gong, J. Chronic neutrophilic leukaemia. J. Clin. Pathol. 68, 680-684 (2015)

67. Erber, W. N. \& Reilly, J. T. Chronic neutrophilic leukemia with plasma cell dyscrasia: friends or relatives? Leuk. Lymphoma 55, 240-242 (2014).

68. Nagai, M. et al. Granulocyte-colony stimulating factor concentrations in a patient with plasma cell dyscrasia and clinical features of chronic neutrophilic leukaemia. J. Clin. Pathol, 49, 858-860 (1996).

69. Kohmura, K., Miyakawa, Y., Kameyama, K., Kizaki, M. \& Ikeda, Y. Granulocyte colony stimulating factor-producing multiple myeloma associated with neutrophilia. Leuk. Lymphoma 45, 1475-1479 (2004).

70. Saitoh, H. \& Shibata, A. Serum G-CSF levels in primary myelofibrosis and chronic neutrophilic leukemia as estimated by the highly sensitive chemiluminescence enzyme immunoassay (CLEIA). Leuk. Lymphoma 22, 515-517 (1996)

71. Usuda, H., Naito, M., Ohyach, K. \& lizumi, T. A case of multiple myeloma producing granulocyte colony-stimulating factor. Pathol. Int. 47, 866-869 (1997)

72. Kusaba, N. et al. [Granulocyte-colony stimulating factor-producing myeloma with clinical manifestations mimicking chronic neutrophilic leukemia]. Rinsho Ketsueki 45, 228-232 (2004).

73. Rodríguez-Medina, C. S.-Z. B., Gómez-Casares, M. T., Lemes Castellano, A. \& López-Jorge, C. E. et al. Ectopic G-Csf production by malignant plasma cells in patients with diagnostic criteria of chronic neutrophilic leukemia. J. Bone Marrow Res. 1, 111 (2013)

74. Sebasky, M. M., Gupta, P. \& Filice, G. A. Elevated granulocyte colonystimulating factor, non-infectious leukocytosis and fevers in a patient with multiple myeloma. J. Gen. Intern. Med. 23, 2134-2135 (2008).

75. Ito, T. et al. Chronic neutrophilic leukemia associated with monoclonal gammopathy of undetermined significance. Acta Haematol. 95, 140-143 (1996)

76. Pardanani, A. et al. CSF3R T6181 is a highly prevalent and specific mutation in chronic neutrophilic leukemia. Leukemia 27, 1870-1873 (2013).

77. Nedeljkovic, M., He, S., Szer, J. \& Juneja, S. Chronic neutrophilia associated with myeloma: is it clonal? Leuk. Lymphoma 55, 439-440 (2014).

78. Blombery, P. et al. Plasma cell neoplasm associated chronic neutrophilic leukemia with membrane proximal and truncating CSF3R mutations. Leuk. Lymphoma 55, 1661-1662 (2014). 
79. Stevens, B. et al. Clonality of neutrophilia associated with plasma cell neoplasms: report of a SETBP1 mutation and analysis of a single institution series. Leuk. Lymphoma 57, 927-934 (2016).

80. Pascucci, M., Dorion, P., Makary, A. \& Froberg, M. K. Chronic neutrophilic leukemia evolving from a myelodysplastic syndrome. Acta Haematol. 98, 163-166 (1997).

81. Ito, K., Usuki, K., Iki, S. \& Urabe, A. Chronic neutrophilic leukemia associated with chronic lymphocytic leukemia. Int. J. Hematol. 68, 87-94 (1998).

82. Wang, R. et al. Nephrotic syndrome related to chronic neutrophilic leukemia. Intern. Med. 53, 2505-2509 (2014).

83. Kwong, Y. \& Cheng, G. Clonal nature of chronic neutrophilic leukemia [letter] [see comments]. Blood 82, 1035-1036 (1993).

84. Bohm, J., Kock, S., Schaefer, H. E. \& Fisch, P. Evidence of clonality in chronic neutrophilic leukaemia. J. Clin. Pathol. 56, 292-295 (2003).

85. Froberg, M. K., Brunning, R. D., Dorion, P., Litz, C. E. \& Torlakovic, E. Demonstration of clonality in neutrophils using FISH in a case of chronic neutrophilic leukemia. Leukemia 12, 623-626 (1998).

86. Didonato, $\mathrm{C}$. et al. Chronic neutrophilic leukemia - description of a new case with karyotypic abnormalities. Am. J. Clin. Pathol. 85, 369-371 (1986).

87. Matano, S. et al. Deletion of the long arm of chromosome 20 in a patient with chronic neutrophilic leukemia: Cytogenetic findings in chronic neutrophilic leukemia. Am. J. Hematol. 54, 72-75 (1997).

88. Piliotis, E., Kutas, G. \& Lipton, J. H. Allogeneic bone marrow transplantation in the management of chronic neutrophilic leukemia. Leuk. Lymphoma $\mathbf{4 3}$ 2051-2054 (2002).

89. Elliott, M. A. Chronic neutrophilic leukemia: a contemporary review. Curr. Hematol. Rep. 3, 210-217 (2004)

90. Mc Lornan, D., Percy, M., Jones, A., Cross, N. \& Mc Mullin, M. Chronic neutrophilic leukemia with an associated V617F JAK2 tyrosine kinase mutation. Haematologica 90, 1696-1697 (2005).

91. Metcalf, D. The granulocyte-macrophage colony-stimulating factors. Science 229, 16-22 (1985).

92. Lieschke, G. J. et al. Mice lacking granulocyte colony-stimulating factor have chronic neutropenia, granulocyte and macrophage progenitor cell deficiency, and impaired neutrophil mobilization. Blood 84, 1737-1746 (1994)

93. Liu, F., Wu, H. Y., Wesselschmidt, R., Kornaga, T. \& Link, D. C. Impaired production and increased apoptosis of neutrophils in granulocyte colonystimulating factor receptor-deficient mice. Immunity 5, 491-501 (1996).

94. Dong, F. et al. Distinct cytoplasmic regions of the human granulocyte colony-stimulating factor receptor involved in induction of proliferation and maturation. Mol. Cell. Biol. 13, 7774-7781 (1993).

95. Corey, S. J. et al. Requirement of Src kinase Lyn for induction of DNA synthesis by granulocyte colony-stimulating factor. J. Biol. Chem. 273, 3230-3235 (1998).

96. Corey, S. J. et al. Granulocyte colony-stimulating factor receptor signaling involves the formation of a three-component complex with Lyn and Syk protein-tyrosine kinases. Proc. Natl Acad. Sci. USA 91, 4683-4687 (1994).

97. Beekman, R. \& Touw, I. P. G-CSF and its receptor in myeloid malignancy. Blood 115, 5131-5136 (2010)

98. Germeshausen, M., Ballmaier, M. \& Welte, K. Incidence of CSF3R mutations in severe congenital neutropenia and relevance for leukemogenesis: results of a long-term survey. Blood 109, 93-99 (2007).

99. Dale, D. C. \& Link, D. C. The many causes of severe congenital neutropenia. N. Engl. J. Med. 360, 3-5 (2009).

100. Dong, F. et al. Identification of a nonsense mutation in the granulocytecolony-stimulating factor receptor in severe congenital neutropenia. Proc. Natl Acad. Sci. USA 91, 4480-4484 (1994).

101. Dong, F. et al. Mutations in the gene for the granulocyte colony-stimulatingfactor receptor in patients with acute myeloid leukemia preceded by severe congenital neutropenia. N. Engl. J. Med. 333, 487-493 (1995).

102. Welte, K. \& Boxer, L. A. Severe chronic neutropenia: pathophysiology and therapy. Semin. Hematol. 34, 267-278 (1997).

103. Beekman, R. et al. Sequential gain of mutations in severe congenital neutropenia progressing to acute myeloid leukemia. Blood 119, 5071-5077 (2012).

104. Zeidler, C., Germeshausen, M., Klein, C. \& Welte, K. Clinical implications of ELA2-, HAX1-, and G-CSF-receptor (CSF3R) mutations in severe congenital neutropenia. Br. J. Haematol. 144, 459-467 (2009)

105. Vandenberghe, P. \& Beel, K. Severe congenital neutropenia, a genetically heterogeneous disease group with an increased risk of AML/MDS. Pediatr. Rep. 3, e9 (2011).
106. Hunter, M. G. \& Avalos, B. R. Granulocyte colony-stimulating factor receptor mutations in severe congenital neutropenia transforming to acute myelogenous leukemia confer resistance to apoptosis and enhance cell survival. Blood 95, 2132-2137 (2000).

107. Kunter, G., Woloszynek, J. R. \& Link, D. C. A truncation mutant of Csf3r cooperates with PML-RARalpha to induce acute myeloid leukemia in mice. Exp. Hematol. 39, 1136-1143 (2011).

108. Liu, F. et al. Csf3r mutations in mice confer a strong clonal HSC advantage via activation of Stat5. J. Clin. Invest. 118, 946-955 (2008).

109. Plo, I. et al. An activating mutation in the CSF3R gene induces a hereditary chronic neutrophilia. J. Exp. Med. 206, 1701-1707 (2009).

110. Bernard, T., Gale, R. E. \& Linch, D. C. Analysis of granulocyte colony stimulating factor receptor isoforms, polymorphisms and mutations in normal haemopoietic cells and acute myeloid leukaemia blasts. Br. J. Haematol. 93, 527-533 (1996).

111. Forbes, L. V. et al. An activating mutation in the transmembrane domain of the granulocyte colony-stimulating factor receptor in patients with acute myeloid leukemia. Oncogene 21, 5981-5989 (2002).

112. Maxson, J. E. et al. The colony-stimulating factor 3 receptor T640N mutation is oncogenic, sensitive to JAK inhibition, and mimics T618I. Clin. Cancer Res. 22 757-764 (2016)

113. Zhang, $\mathrm{H}$. et al. Unpaired extracellular cysteine mutations of CSF3R mediate gain or loss of function. Cancer Res. 77, 4258-4267 (2017).

114. Maxson, J. E. et al. CSF3R mutations have a high degree of overlap with CEBPA mutations in pediatric AML. Blood 127, 3094-3098 (2016).

115. Zhang, $\mathrm{H}$. et al. Characterization of the leukemogenic potential of distal cytoplasmic CSF3R truncation and missense mutations. Leukemia $\mathbf{3 1}$ 2752-2760 (2017)

116. Maxson, J. E. et al. Ligand independence of the T618I mutation in the colonystimulating factor 3 receptor (CSF3R) protein results from loss of O-linked glycosylation and increased receptor dimerization. J. Biol. Chem. 289, 5820-5827 (2014)

117. Aarts, L. H., Roovers, O., Ward, A. C. \& Touw, I. P. Receptor activation and 2 distinct $\mathrm{COOH}$-terminal motifs control G-CSF receptor distribution and internalization kinetics. Blood 103, 571-579 (2004).

118. Hortner, M. et al. Suppressor of cytokine signaling-3 is recruited to the activated granulocyte-colony stimulating factor receptor and modulates its signal transduction. J. Immunol. 169, 1219-1227 (2002).

119. Dong, F., Qiu, Y., Yi, T., Touw, I. P. \& Larner, A. C. The carboxyl terminus of the granulocyte colony-stimulating factor receptor, truncated in patients with severe congenital neutropenia/acute myeloid leukemia, is required for $\mathrm{SH}_{2}$ containing phosphatase-1 suppression of Stat activation. J. Immunol. 167, 6447-6452 (2001)

120. Fleischman, A. G. et al. The CSF3R T618I mutation causes a lethal neutrophilic neoplasia in mice that is responsive to therapeutic JAK inhibition. Blood $\mathbf{1 2 2}$ 3628-3631 (2013)

121. Gotlib, J., Maxson, J. E., George, T. I. \& Tyner, J. W. The new genetics of chronic neutrophilic leukemia and atypical CML: implications for diagnosis and treatment. Blood 122, 1707-1711 (2013).

122. Maxson, J. E. \& Tyner, J. W. Genomics of chronic neutrophilic leukemia. Blood 129, 715-722 (2017).

123. Rohrabaugh, $\mathrm{S}$. et al. Enhanced MAPK signaling is essential for CSF3Rinduced leukemia. Leukemia 31, 1770-1778 (2017).

124. Beekman, R., Valkhof, M., van Strien, P., Valk, P. J. \& Touw, I. P. Prevalence of a new auto-activating colony stimulating factor 3 receptor mutation (CSF3RT595I) in acute myeloid leukemia and severe congenital neutropenia. Haematologica 98, e62-e63 (2013).

125. Wang, S. A. et al. Atypical chronic myeloid leukemia is clinically distinct from unclassifiable myelodysplastic/myeloproliferative neoplasms. Blood 123, 2645-2651 (2014)

126. Cui, Y. et al. CSF3R, SETBP1 and CALR mutations in chronic neutrophilic leukemia. J. Hematol. Oncol. 7, 77 (2014).

127. Lasho, T. L., Elliott, M. A., Pardanani, A. \& Tefferi, A. CALR mutation studies in chronic neutrophilic leukemia. Am. J. Hematol. 89, 450 (2014).

128. Cristóbal, I. et al. SETBP1 overexpression is a novel leukemogenic mechanism that predicts adverse outcome in elderly patients with acute myeloid leukemia. Blood 115, 615-625 (2010).

129. Piazza, R. et al. Recurrent SETBP1 mutations in atypical chronic myeloid leukemia. Nat. Genet. 45, 18-24 (2013).

130. Makishima, H. et al. Somatic SETBP1 mutations in myeloid malignancies. Nat. Genet. 45, 942-946 (2013). 
131. Tefferi, A., Elliott, M. \& Pardanani, A. Chronic neutrophilic leukemia: novel mutations and their impact on clinical practice. Curr. Opin. Hematol. 22 171-176 (2015)

132. Cui, Y. J. et al. TThe clinical characteristics, gene mutations and prognosis of chronic neutrophilic leukemia]. Zhonghua. Xue. Ye. Xue. Za. Zhi. 38, 28-32 (2017).

133. Lasho, T. L. et al. Chronic neutrophilic leukemia with concurrent CSF3R and SETBP1 mutations: single colony clonality studies, in vitro sensitivity to JAK inhibitors and lack of treatment response to ruxolitinib. Leukemia $\mathbf{2 8}$ 1363-1365 (2014).

134. Ammatuna, E. et al. Atypical chronic myeloid leukemia with concomitant CSF3R T618I and SETBP1 mutations unresponsive to the JAK inhibitor ruxolitinib. Ann. Hematol. 94, 879-880 (2015).

135. Nooruddin, Z et al. Changes in allele frequencies of CSF3R and SETBP1 mutations and evidence of clonal evolution in a chronic neutrophilic leukemia patient treated with ruxolitinib. Haematologica 102, e207-e209 (2017).

136. Shou, L. H. et al. Prognostic significance of SETBP1 mutations in myelodysplastic syndromes, chronic myelomonocytic leukemia, and chronic neutrophilic leukemia: a meta-analysis. PLOS ONE 12, e0171608 (2017).

137. Steensma, D. P. et al. The JAK2 V617F activating tyrosine kinase mutation is an infrequent event in both "atypical" myeloproliferative disorders and myelodysplastic syndromes. Blood 106, 1207-1209 (2005).

138. Kako, S. et al. Early relapse of JAK2 V617F-positive chronic neutrophilic leukemia with central nervous system infiltration after unrelated bone marrow transplantation. Am. J. Hematol. 82, 386-390 (2007).

139. Imashuku, S. et al. Rituximab for managing acquired hemophilia A in a case of chronic neutrophilic leukemia with the JAK2 kinase V617F mutation. J. Blood Med. 3, 157-161 (2012)

140. Zhang, X., Pan, J. \& Guo, J. Presence of the JAK2 V617F mutation in a patient with chronic neutrophilic leukemia and effective response to interferon alpha-2b. Acta Haematol. 130, 44-46 (2013).

141. Gajendra, S. et al. Chronic Neutrophilic Leukemia with V617F JAK2 Mutation. Indian J. Hematol. Blood Transfus. 30, 139-142 (2014).

142. Lea, N. C. et al. Presence of JAK2 V617F tyrosine kinase mutation as a myeloid-lineage-specific mutation in chronic neutrophilic leukaemia. Leukemia 20, 1324-1326 (2006).

143. Lee, J.-H., Ha, J.-S., Ryoo, N.-H., Jeon, D.-S. \& Kim, J.-R. A case of acute myeloid leukemia transformed from JAK2 V617F-positive chronic neutrophilic leukemia. Lab Med Online 2, 101-104 (2012)

144. Ortiz-Cruz, K., Amog-Jones, G. \& Salvatore, J. Chronic neutrophilic leukemia with JAK2 gene mutation. Community Oncol. 9, 127-131 (2012).

145. Thiele, J. Philadelphia chromosome-negative chronic myeloproliferative disease. Am. J. Clin. Pathol. 132, 261-280 (2009).

146. Sugino, K. et al. Miliary tuberculosis associated with chronic neutrophilic leukemia. Intern. Med. 48, 1283-1287 (2009).

147. Senin, A. et al. [Molecular characterization of atypical chronic myeloid leukemia and chronic neutrophilic leukemia]. Med. Clin. 144, 487-490 (2015).

148. Dao, K. H. \& Tyner, J. W. What's different about atypical CML and chronic neutrophilic leukemia? Hematol. Am. Soc. Hematol. Educ. Program. 2015 264-271 (2015)

149. Mohr, F., Dohner, K., Buske, C. \& Rawat, V. P. TET genes: new players in DNA demethylation and important determinants for stemness. Exp. Hematol. 39 272-281 (2011)

150. Dao, K. T., Tyner, J. W. \& Gotlib, J. Recent progress in chronic neutrophilic leukemia and atypical chronic myeloid leukemia. Curr. Hematol. Malig. Rep. 12, 432-441 (2017)
151. Busque, L. et al. Recurrent somatic TfET2 mutations in normal elderly individuals with clonal hematopoiesis. Nat. Genet. 44, 1179-1181 (2012).

152. Klampfl, T. et al. Somatic mutations of calreticulin in myeloproliferative neoplasms. N. Engl. J. Med. 369, 2379-2390 (2013).

153. Nangalia, J. et al. Somatic CALR mutations in myeloproliferative neoplasms with nonmutated JAK2. N. Engl. J. Med. 369, 2391-2405 (2013).

154. Menezes, J. et al. CSF3R T618l co-occurs with mutations of splicing and epigenetic genes and with a new PIM3 truncated fusion gene in chronic neutrophilic leukemia. Blood. Cancer J. 3, e158 (2013).

155. Makishima, $\mathrm{H}$. et al. Mutations in the spliceosome machinery, a novel and ubiquitous pathway in leukemogenesis. Blood 119, 3203-3210 (2012).

156. Langabeer, S. E., Haslam, K., Kelly, J. et al. Targeted next-generation sequencing identifies clinically relevant mutations in patients with chronic neutrophilic leukemia at diagnosis and blast crisis. Clin. Transl. Oncol. (2017). https://doi.org/10.1007/s12094-017-1722-2

157. Meyer, S. et al. Successful alpha-2b-interferon therapy for chronic neutrophilic leukemia. Am. J. Hematol. 43, 307-309 (1993).

158. Menezes, J. \& Cigudosa, J. C. Chronic neutrophilic leukemia: a clinical perspective. OncoTargets Ther. 8, 2383-2390 (2015)

159. Lorente, J. A. et al. A case of chronic neutrophilic leukemia with original chromosomal abnormalities. Eur. J. Haematol. 41, 285-288 (1988).

160. Elliott, M. A., Dewald, G. W., Tefferi, A. \& Hanson, C. A. Chronic neutrophilic leukemia (CNL): a clinical, pathologic and cytogenetic study. Leukemia 15, 35-40 (2001).

161. Goto, H., Hara, T., Tsurumi, H., Tanabashi, S. \& Moriwaki, H. Chronic neutrophilic leukemia with congenital Robertsonian translocation successfully treated with allogeneic bone marrow transplantation in a young man. Intern. Med. 48, 563-567 (2009).

162. Lee, S. E. et al. T6181-mutated colony stimulating factor 3 receptor in chronic neutrophilic leukemia and chronic myelomonocytic leukemia patients who underwent allogeneic stem cell transplantation. Ann. Lab Med. 35, 376-378 (2015)

163. Langabeer, S. E., McCarron, S. L., Haslam, K., O'Donovan, M. T. \& Conneally, E. The CSF3R T618I mutation as a disease-specific marker of atypical CML post allo-SCT. Bone Marrow Transplant. 49, 843-844 (2014).

164. Cervantes, F. et al. Three-year efficacy, safety, and survival findings from COMFORT-II, a phase 3 study comparing ruxolitinib with best available therapy for myelofibrosis. Blood 122, 4047-4053 (2013).

165. Vannucchi, A. M. Ruxolitinib versus standard therapy for the treatment of polycythemia vera. N. Engl. J. Med. 372, 1670-1671 (2015).

166. Stahl, M. et al. Clinical response to ruxolitinib in CSF3R T618-mutated chronic neutrophilic leukemia. Ann. Hematol. 95, 1197-1200 (2016)

167. Dao, K. H. et al. Significant clinical response to JAK1/2 inhibition in a patient with CSF3R-T618l-positive atypical chronic myeloid leukemia. Leuk. Res. Rep. 3, 67-69 (2014).

168. Gunawan, A. S. et al. Ruxolitinib, a potent JAK1/JAK2 inhibitor, induces temporary reductions in the allelic burden of concurrent CSF3R mutations in chronic neutrophilic leukemia. Haematologica 102, e238-e240 (2017).

169. Koppikar, P. et al. Heterodimeric JAK-STAT activation as a mechanism of persistence to JAK2 inhibitor therapy. Nature 489, 155-159 (2012).

170. Borthakur, G. et al. Activity of the oral mitogen-activated protein kinase kinase inhibitor trametinib in RAS-mutant relapsed or refractory myeloid malignancies. Cancer 122, 1871-1879 (2016).

171. Khanna, V. et al. Durable disease control with MEK inhibition in a patient with NRAS-mutated atypical chronic myeloid leukemia. Cureus 7, e414 (2015). 